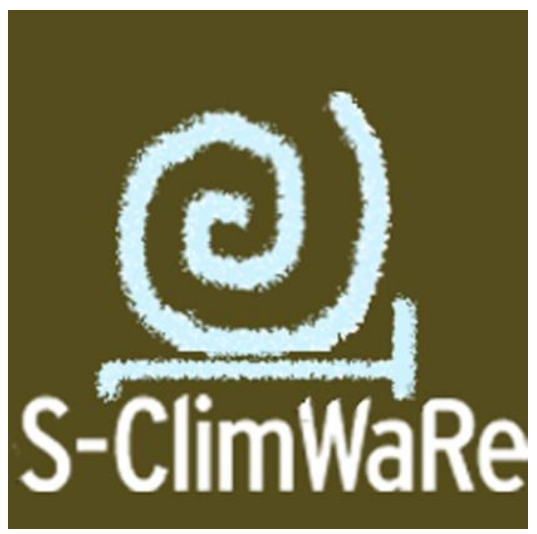

\title{
Sistema estadístico de predicción estacional para la gestión de los embalses en España
}

\author{
Nota técnica 21 de AEMET
}

2016

\author{
José Voces Aboy \\ Eroteida Sánchez García \\ Beatriz Navascués Fernández-Victorio \\ Federico Franco Manzano \\ Ernesto Rodríguez Camino
}




\section{S-ClimWaRe}

\section{Servicios climáticos en apoyo a la gestión de los embalses en España}

Autores: José Voces Aboy, Eroteida Sánchez García, Beatriz Navascués Fernández-Victorio, Federico Franco Manzano, Ernesto Rodríguez Camino (2016)

Aviso Legal: los contenidos de esta publicación podrán ser reutilizados, citando la fuente y la fecha, en su caso, de la última actualización

\section{Edita:}

(c) Ministerio de Agricultura, Alimentación y Medio Ambiente Agencia Estatal de Meteorología Madrid, 201

Catálogo de Publicaciones de la Administración General del Estado: http://publicacionesoficiales.boe.es/

https://doi.org/10.31978/281-16-003-1

Agencia Estatal de Meteorología (AEMET)

C/ Leonardo Prieto Castro, 8

28040 Madrid

http://www.aemet.es/

@Aemet_Esp 


\section{Resumen}

Se presenta un servicio climático desarrollado a medida para apoyar la gestión de los embalses. Estos trabajos se han diseñado para contribuir a la implementación del Marco Mundial para los Servicios Climáticos (MMSC) en España, y forman parte de un "caso de estudio" del proyecto europeo FP7 EUPORIAS (EUropean Provision Of Regional Impacts Assessments on Seasonal and decadal timescales) denominado S-ClimWaRe (Seasonal Climate predictions in support of Water Reservoirs management in Spain). S-ClimWaRe tiene como objetivo desarrollar un sistema completo de predicción del volumen de entrada y de previsión del estado del embalse a escala estacional, que pueda ser utilizado por los gestores de los recursos hídricos en sus procesos de toma de decisiones. Este documento describe la componente de predicción estacional del volumen de entrada (o aportaciones) y de precipitación, que ha sido desarrollada por AEMET.

Tomando como base la importante influencia de la Oscilación del Atlántico Norte (NAO) en la variabilidad hidrológica en amplias zonas de España, y teniendo en cuenta que la NAO está estadísticamente asociada a la evolución de la cobertura de nieve en las zonas continentales del Hemisferio Norte durante el mes de octubre, se ha desarrollado un sistema probabilístico de predicción estacional basado en métodos empíricos que hace uso de datos de observación hidrológicos y meteorológicos. El sistema se ha evaluado de forma retrospectiva en 4 embalses seleccionados de las cuencas del Duero, Tajo, Ebro y Guadalquivir mediante la realización de varios experimentos que hacen uso de índices de cobertura de nieve diferentes como factor determinante de la posterior NAO invernal. Los experimentos se han evaluado aplicando métricas estándar utilizadas para la verificación objetiva de predicciones deterministas y probabilísticas.

Los resultados obtenidos indican que, en general, las predicciones estacionales de las aportaciones y la precipitación en el trimestre invernal producidas por el sistema desarrollado presentan una pericia significativa en los embalses estudiados de las cuencas del Duero, Tajo y Guadalquivir, especialmente a la hora de discriminar si se esperan periodos secos o húmedos. En el caso del Embalse del Ebro, también se observa cierta pericia para discriminar el tercil húmedo. El sistema desarrollado muestra ser sensible al índice de nieve escogido como predictor de la NAO invernal, consiguiéndose mejores resultados cuando se utilizan productos satelitales de cobertura de nieve de frecuencia diaria para describir la evolución del manto nivoso. 


\section{INDICE}

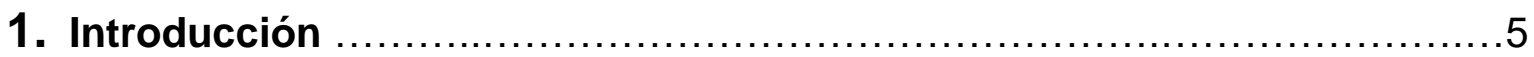

2. Modelo estadístico de predicción estacional de aportaciones a los embalses

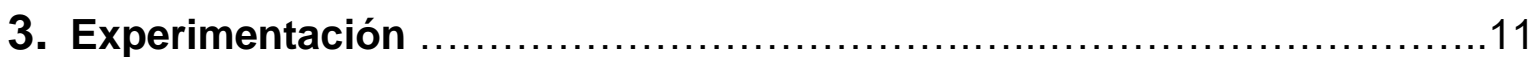

4. Evaluación del sistema de predicción estacional ............................16

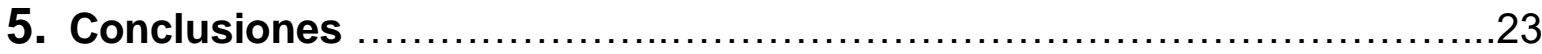





\section{ANEXO I}

Evaluación del sistema "SAI_Eurasia_weekly_ext" para dos periodos de verificación diferentes. 


\section{Introducción}

En la Tercera Conferencia Mundial del Clima celebrada en el año 2009, los jefes de gobierno y altos representantes de los gobiernos de más de 150 países decidieron establecer el Marco Mundial para los Servicios Climáticos. La visión de esta estructura intergubernamental, promovida por las Naciones Unidas y liderada por la Organización Meteorológica Mundial, es la de una sociedad que gestione mejor los riesgos y las oportunidades vinculados a la variabilidad del clima y el cambio climático, desarrollando e incorporando información y predicciones climáticas con base científica en la planificación, en las políticas, y en las actividades prácticas. El MMSC define los servicios climáticos como el suministro de información climática que ayude a la toma de decisiones, tanto de las personas como de las organizaciones. Por ello, uno de los cinco pilares en los que se sustenta es la llamada Plataforma Interfaz de Usuarios, cuyo objetivo es la interacción regular y sistemática entre los usuarios y los proveedores de servicios climáticos. Tanto la propia ciencia del clima como la aplicación de la información climática a la toma de decisiones son cuestiones muy complejas, por lo que se hace necesario un esfuerzo multidisciplinar, en el que la clave del éxito son las alianzas y la explotación de las sinergias existentes.

El proyecto europeo FP7 EUPORIAS trabaja en el desarrollo de sistemas de previsión de impactos a escala estacional y decadal para el apoyo a la toma de decisiones dependientes del clima, siguiendo las directrices del MMSC, y fomentando el desarrollo de los servicios climáticos en sectores clave, incluyendo el agua, la energía, la salud, el transporte, la agricultura y el turismo. AEMET participa en el proyecto EUPORIAS, junto con otras 23 instituciones, en especial en los servicios de predicción estacional en el sector de los recursos hídricos.

En el mes de marzo de 2014, dentro de las actividades de implementación en España del MMSC y en coordinación con el proyecto europeo FP7 EUPORIAS, se celebró en la sede central de la Agencia Estatal de Meteorología un Taller de Trabajo sobre la Utilización de las Predicciones Climáticas Estacionales para la Mejora de la Gestión del Agua. La activa participación de la Dirección General del Agua y de varias Confederaciones Hidrográficas contribuyó de forma decisiva al éxito de este evento. Los participantes acordaron dar continuidad a este tipo de interacción y se dio prioridad a la celebración de encuentros específicos destinados a conocer los procesos de toma de decisión de los usuarios y extender y mejorar el conocimiento sobre las predicciones estacionales. También se identificó como proyecto piloto demostrativo del valor de las predicciones climáticas estacionales en la toma de decisiones, el desarrollo de un servicio climático en apoyo a la gestión de embalses.

Tras la celebración del taller, la Dirección General del Agua y AEMET impulsaron la formación de un grupo de trabajo multidisciplinar para el desarrollo de esta experiencia piloto. Al grupo se incorporaron representantes de las Confederaciones del Duero, Tajo y Ebro, así como CETaqua y la Universidad Politécnica de Valencia. El grupo de trabajo redactó y acordó un proyecto experimental que se ha conformado como un caso de estudio de EUPORIAS, bajo el nombre de S-ClimWaRe (Seasonal Climate predictions in support of Water Reservoirs management in Spain). Representantes de la Confederación del Mlño-Sil se han unido también al proyecto recientemente. Los participantes en esta experiencia pretenden que sea la base para seguir avanzando en el uso de las predicciones climáticas estacionales para la gestión de los recursos hídricos mediante proyectos de I+D+i de alcance nacional y europeo.

En S-ClimWaRe se han seleccionado 4 embalses no regulados, o apenas regulados aguas arriba, de las cuencas del Duero, Tajo, Ebro y Guadalquivir en los que se está probando la utilización de las predicciones climáticas estacionales (Pouget et al., 2015). El proyecto combina datos de observación hidrológicos y meteorológicos, el sistema estadístico de predicción estacional de aportaciones que se describe en este documento, y un modelo de previsión de riesgos de los embalses, SIMRISK, que fue desarrollado por la Universidad Politécnica de Valencia (Andreu et al., 1996). En este proyecto, SIMRISK está siendo adaptado para utilizar como entrada las predicciones probabilísticas de aportaciones a los embalses que suministra AEMET.

El caso de estudio se apoya en sistemas ya probados con éxito en otros países, fundamentalmente de Asia y Norteamérica, basados en la predecibilidad del clima a escala estacional que proporciona el fenómeno de El Niño. EI IRI (International Research Institute for Climate and Society) de EEUU, ha participado activamente en estos desarrollos (Brown et al., 2010). Sus trabajos están siendo una referencia para esta experiencia pionera en España. 
Sin embargo, en términos generales, la predecibilidad a escala estacional para España es baja, y consiguientemente la pericia de los modelos. Ello hace que, de momento, en nuestro país, el uso de las predicciones estacionales sea bastante limitado. No obstante, estas predicciones pueden tener una mayor fiabilidad para unos periodos del año, unas zonas, o una variable. Es lo que se conoce como "ventana de oportunidad" para la predicción estacional.

Cuando examinamos las herramientas más complejas de las que disponemos en el campo de la predicción en estas escalas, los modelos numéricos de circulación general océano-atmósfera, se observa (Sánchez et al., 2014) que en España, aunque existen algunas ventanas de oportunidad (como la temperatura en verano), a día de hoy, no presentan por norma general una gran habilidad para predecir la precipitación. En ese sentido, y de cara a desarrollar un sistema de predicción de las aportaciones a los embalses, en este proyecto se ha optado por hacer uso del hecho de que la precipitación en invierno en amplias zonas de España está muy relacionada con la Oscilación del Atlántico Norte (NAO), un conocido patrón dominante de la variabilidad del clima en la zona del Atlántico Norte (ver por ejemplo, Rodríguez-Puebla et al. 1998). Sin embargo, la utilidad de esta relación depende críticamente de la pericia para predecir la NAO. La mayoría de los modelos de circulación general todavía muestran un poder predictivo limitado para la NAO en las escalas de tiempo estacionales, con la excepción de algunos buenos resultados solo obtenidos recientemente (Scaife et al. 2014).

Por otro lado, Cohen y Jones (2011) muestran que la Oscilación del Ártico (AO) en invierno (de la que la NAO es una manifestación regional) está estadísticamente asociada al avance de la cobertura de nieve en Eurasia durante el mes de octubre (véase Figura 1), y definen un índice basado en la medida de satélite de esta magnitud, el Snow Advance Index (SAI).



Figura 1. Serie temporal del SAI diario (en azul) y la AO invernal (en negro) para el periodo 1997/98 a 2010/11. Se ha eliminado la tendencia de ambas series y se muestra la anomalía estandarizada. EI SAI se ha multiplicado por -1 para facilitar la comparación. El coeficiente de correlación es estadísticamente significativo a un nivel $p<0.01$. Fuente: Cohen y Jones (2011)

Haciendo uso de esta relación, a través de la cual se adquiere la capacidad de pronosticar la NAO en invierno, los modelos de aprendizaje estadístico aparecen como una alternativa para el desarrollo de un sistema de predicción de las aportaciones en invierno. En esta línea, en Brands et al. (2012) se pone de manifiesto cómo haciendo uso del SAI apuntado anteriormente, es posible hacer un pronóstico estacional de la precipitación invernal en España con una regresión lineal que utiliza como único predictor el índice de avance de la cobertura de la nieve en octubre (véase Figura 2). 


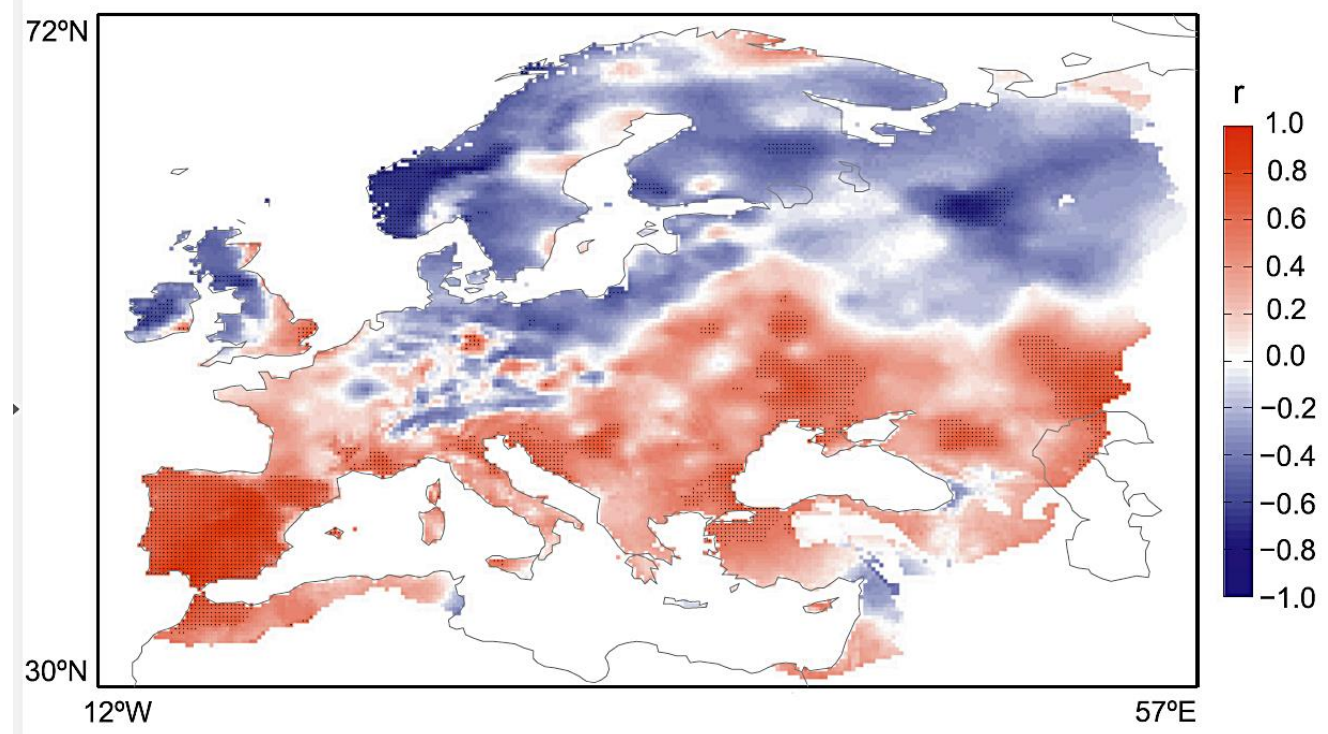

Figura 2. Correlación entre el SAI diario y la precipitación en el trimestre diciembre-enerofebrero. Las correlaciones estadísticamente significativas (con $\alpha=0.05$ ) se han sombreado. Fuente: Brands et al. (2012)

Se dan pues las condiciones para probar un sistema empírico de predicción estacional que pronostique la aportación invernal a un embalse con ciertas garantías.

Los modelos de circulación general océano-atmósfera permiten simular las evoluciones posibles, a partir de una situación inicial conocida, del estado del océano y del resto de componentes del sistema climático. Sin embargo, éstos representan de forma aproximada los procesos físico-químicos que se producen en las componentes del sistema climático y sus interacciones. Tanto la descripción de estos procesos, como la del estado inicial del sistema climático, son imprecisas. Debido a su inherente reducción del problema, los sistemas de predicción estadísticos también tienen una gran incertidumbre. La incertidumbre asociada a las predicciones climáticas producidas con modelos dinámicos o estadísticos se intenta estimar y cuantificar mediante la realización de múltiples simulaciones. Una representación de los posibles estados futuros se materializa realizando diferentes simulaciones con pequeños cambios en un mismo modelo $o$ sistema de predicción, con condiciones iniciales distintas o utilizando diferentes modelos. Por este motivo, a escala climática las predicciones estacionales proporcionan información probabilística: existe no sólo uno sino varios futuros posibles, cuya estadística se puede comparar con la climatología.

En este caso de estudio hemos adoptado una aproximación ensemble de simulaciones para representar la incertidumbre de las predicciones. Uno de los principales retos para el desarrollo de servicios climáticos a medida, como el que estamos explorando en S-ClimWare, es demostrar la utilidad de estas predicciones climáticas estacionales que son de carácter probabilístico. Ello requiere una adaptación de los usuarios, que en general, no están acostumbrados a utilizar sistemas de gestión de riesgos en base a predicciones climáticas probabilísticas en su toma de decisiones. Para facilitar este proceso, en este caso de estudio se sigue una metodología próxima a la utilizada en Gong et al. (2010) y en Brown et al (2010), donde se hace hincapié en cómo afecta la estimación de la incertidumbre en las diferentes componentes del servicio climático de apoyo a la gestión de los embalses.

Cabe señalar que el método estadístico utilizado en este proyecto para el sistema probabilístico de predicción estacional de aportaciones es mucho más sencillo y menos costoso en recursos de computación que los basados en métodos físicos, que aunque utilicen salidas de modelos acoplados océano-atmósfera ya existentes, necesitan de un paso adicional de regionalización y la ejecución posterior de modelos hidrológicos para la predicción del volumen de entrada a los embalses.

La estructura de este documento es la siguiente. En la sección 2 se describe la metodología aplicada para el modelo de predicción de aportaciones y/o precipitación desarrollado. La sección 3 presenta el diseño de los experimentos realizados con el prototipo desarrollado. La evaluación de estos experimentos se trata en la sección 4. Por último, las conclusiones y las futuras líneas de investigación 
se encuentran en la sección 5. En el Anexo I se muestran más detalles sobre la evaluación de los experimentos llevados a cabo.

\section{Modelo estadístico de predicción estacional de aportaciones a los embalses}

\section{Metodologías propuestas por el IRI}

Como se ha apuntado en la introducción, en el desarrollo del sistema de predicción estacional para embalses se ha seguido la propuesta descrita en el manual "Managing Climate Risk in Water Supply Systems", del International Research Institute for Climate and Society, por Brown et al. (2010).

En este documento se justifica la necesidad de modificar la gestión de los recursos hídricos pasando de los actuales sistemas basados en una visión estacionaria del clima a unos que tengan en cuenta la variabilidad climática del entorno. Para ello, sugieren diversos métodos estadísticos de diagnóstico de la variabilidad hidroclimática que está ligada a patrones dominantes conocidos (por ejemplo El Niño, en Asia y Norteamérica, o la NAO en la Península lbérica en invierno). A continuación, Brown et al. (2010) presentan diferentes implementaciones de sistemas de predicción estacional de aportaciones. En los sistemas estadísticos que proponen, el primer paso consiste en encontrar una variable que actúe como predictor de las aportaciones a los embalses (el predictando).

Una vez identificado el predictor, se proponen 3 alternativas para relacionar el predictor con el predictando, y construir el ensemble de simulaciones que dan cuenta de la estimación de la incertidumbre y que constituyen la predicción probabilística:

- Regresión lineal simple.

Se utiliza una relación del tipo:

$$
y_{i}=a x_{i}+b+e_{i}
$$

donde $x_{i}$ es el valor del predictor en el año $i$,

$y_{i}$ es el predictando en el año $i$,

$a$ y $b$ son los parámetros del ajuste,

y $e_{i}$ es el error cometido al hacer el pronóstico del año $i$.

Puede suponerse que los errores $e_{i}$ se distribuyen de acuerdo a una distribución normal de media 0 y desviación estándar $\sigma=\sqrt{ }\left(1 / n \sum e_{1}^{2}\right)$. Mediante una muestra aleatoria de esta distribución gaussiana de errores se obtiene un ensemble de simulaciones equiprobables para cada año i.

- Muestreo de los K vecinos más próximos. Algoritmo K-NN (K Nearest Neighbours)

Una aproximación alternativa a la anterior que no hace ninguna suposición sobre la forma de la función de distribución de probabilidad de los errores consiste en aplicar un algoritmo K-NN (Lall y Sharma, 1996, o Gong et al. 2010).

En este caso, dado el valor del predictor en un año i, $x_{i}$, se busca en la serie histórica de valores del predictando, los valores ocurridos $y_{j} j=1, \ldots, k$, que corresponden a los $k$ valores más cercanos a $x_{i}$. De esta forma, se obtiene un ensemble de $k$ simulaciones equiprobables.

- Clasificación. La última solución propuesta consiste en separar la serie del predictor en varios intervalos y asignar el correspondiente ensemble de predictandos a cada intervalo del predictor. 


\section{Solución adoptada}

Teniendo en cuenta las particularidades que determinan el comportamiento del clima en amplias zonas de España en invierno, el sistema estadístico utilizado por el IRI en Asia y Norteamérica se adapta perfectamente a los objetivos del proyecto sustituyendo la fuente de predecibilidad utilizada para predecir El Niño (la temperatura del agua del mar), por el índice de cobertura de nieve en otoño para la previsión de la NAO invernal.

De esta forma, el módulo de predicción desarrollado consiste en un sistema estadístico para predecir, a partir del valor del índice de nieve observado (disponible a comienzos de noviembre) un ensemble de valores de la aportación en el trimestre diciembre-enero-febrero (DEF) en un embalse determinado.

Con los datos disponibles, en principio se podría utilizar directamente el índice de nieve como predictor de las aportaciones al embalse. Sin embargo, las series existentes de la NAO y de aportaciones son mucho más largas que las de cobertura de nieve. Para poder representar mejor la variabilidad hidroclimática del embalse, la predicción de aportaciones se realiza en los dos pasos: primero se pronostica el estado de la NAO, y a partir de éste se predice la aportación al embalse o la precipitación. Al utilizar este método, asumimos que el modelo de predicción de la NAO puede ser sintonizado con unas series más cortas de observaciones de cobertura de nieve e índices de la NAO. La ejecución de la predicción en estas dos etapas tiene sentido desde el punto de vista físico. Cohen (2011) propone un modelo dinámico conceptual para explicar cómo la cobertura de nieve en otoño en Siberia modifica la circulación en la troposfera y estratosfera en el invierno siguiente, y afecta a la Oscilación del Ártico, y por tanto a la fase de la NAO. Como se ha mencionado en la introducción, la influencia de la NAO en la precipitación en la Península Ibérica ha sido demostrada por muchos autores (ver, p.ej., RodríguezFonseca y Rodríguez-Puebla 2010).

Por tanto, el sistema de predicción consiste en los dos pasos siguientes:

\section{Pronóstico de la NAO en el trimestre DEF a partir del índice de nieve}

En una primera etapa, el índice de variabilidad de la cobertura de la nieve observada en otoño se utiliza para pronosticar un ensemble de la NAO promedio del próximo trimestre DEF.

Para ello, además del índice de la nieve observada el trimestre anterior al que se quiere predecir, se utilizan los datos del período común disponible de las series de datos observados del índice de nieve y de la NAO(DEF).

La relación entre la NAO y uno de los índices de nieve utilizados, el SAI semanal, se representa en la Figura 3

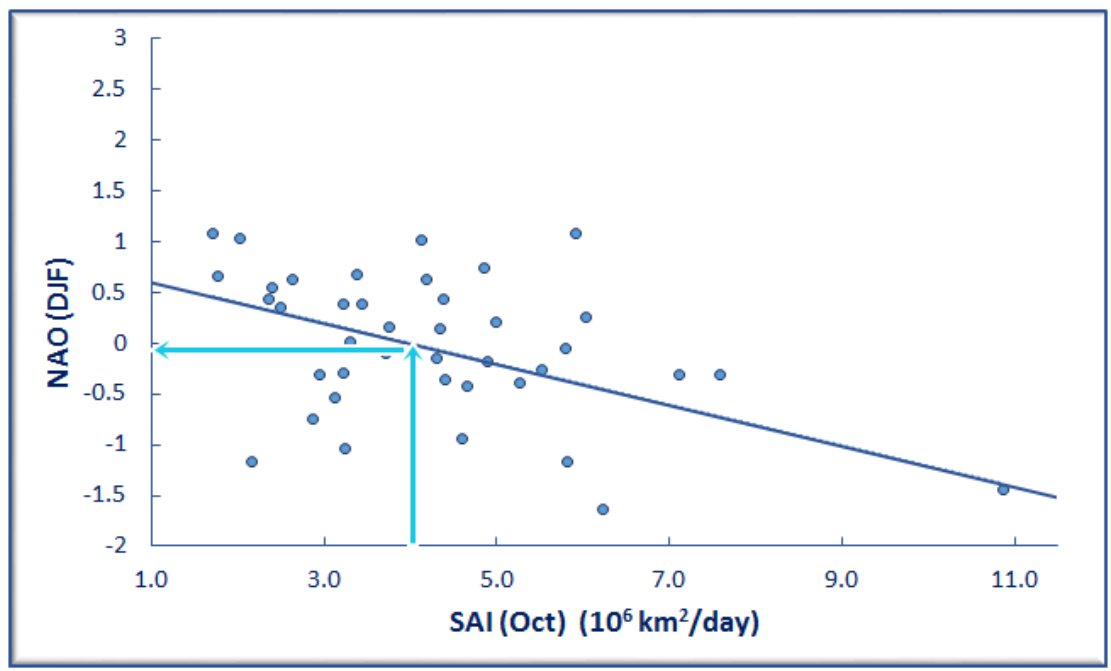

Figura 3. Ajuste de mínimos cuadrados entre la NAO(DEF) y el SAl semanal

Cada valor del SAI observado en otoño permite predecir la NAO(DEF) mediante una regresión lineal simple, en la que los coeficientes del ajuste han sido calculados sin tener en cuenta los 
valores del predictor y del predictando del año en el que se realiza el pronóstico. La estimación de la incertidumbre de este pronóstico se obtiene haciendo una validación cruzada de esta predicción determinista dejando el año en cuestión fuera de la regresión, y calculando la raíz cuadrada del promedio del cuadrado de los errores cometidos. A continuación, se genera una distribución normal sintética (véase Figura 4) cuya media será el valor previsto de la NAO(DEF) por la regresión lineal, y cuya desviación estándar será la estimación del error anteriormente calculada.



Figura 4. Distribución normal sintética (línea) y muestreo de los valores de la NAO sobre la distribución (histograma).

Sobre esta distribución se toman m muestras para obtener un ensemble de valores previstos de la $\mathrm{NAO}(\mathrm{DEF})$ que estima la incertidumbre de este modelo de predicción.

\section{Pronóstico de la aportación(DEF) a partir de la $N A O(D E F)$}

En un segundo paso, para cada valor de la muestra de la NAO(DEF) se realiza el pronóstico de la aportación al embalse en el trimestre DEF. En este caso, se ha optado por utilizar un algoritmo KNN para tener una estimación de la incertidumbre en el pronóstico. Se evita así la suposición de la distribución normal de los errores, y permite tener en cuenta que la dispersión de los datos de aportaciones depende de la fase de la NAO. La existencia de una serie larga de datos de volumen de entrada a los embalses facilita la utilización de esta aproximación. Se obtienen, por tanto, $\mathrm{k}$ valores de aportaciones por cada valor de la NAO.

En la Figura 5 se muestra un ejemplo para el embalse de la Cuerda del Pozo con k = 4 .



Figura 5. Ajuste por mínimos cuadrados de la recta de regresión entre la NAO(DEF) y las Aportaciones(DEF). También se muestran 2 casos de aplicación del algoritmo KNN con $\mathrm{k}=4$ (rectángulos verticales en azul claro). 
De esta forma, se obtiene finalmente un ensemble de predicción de aportaciones formado por $m^{*} k$ miembros.

La elección de los valores para $m$ (el número de muestras tomadas para la NAO) y $k$ (el número de vecinos en las aportaciones) se ha hecho en base a que representen la variabilidad del sistema y minimicen los errores cometidos. El valor de $m$ se ha escogido empíricamente para conseguir que la distribución de los valores de la NAO se aproxime a la distribución gaussiana que se muestrea; en este caso $m=500$ parece un valor adecuado. Una vez fijado el valor de $m$, el valor de $k$ se ha elegido evaluando la habilidad del sistema completo para diferentes valores de $k$. En este caso, el valor seleccionado empíricamente ha sido $k=3$. Es de destacar que para un entorno de los valores elegidos, la habilidad del sistema no varía demasiado, lo que indica que el sistema está dotado de una cierta robustez.

El ensemble de aportaciones generado está, por tanto, compuesto por 1500 valores de aportaciones tomados de la serie histórica del embalse en cuestión. Es decir, está formado por sucesos que ya han ocurrido en el pasado. Cada suceso tendrá una frecuencia asociada, el número de veces que aparece en el ensemble de predicción. Esta frecuencia está directamente relacionada con la probabilidad de esa aportación.

El modelo desarrollado es totalmente aplicable a la predicción estacional de la precipitación, sin más que sustituir la serie de valores observados de aportaciones al embalse por el de precipitación en el segundo paso de predicción.

Los datos brutos del ensemble generado por el sistema de predicción no son muy adecuados para ser trasladados directamente a los responsables de la gestión del embalse, sin embargo sí lo son como entrada de un sistema de apoyo a la toma de decisiones que pueden utilizar los usuarios finales. Una forma más apropiada de presentar el pronóstico a los usuarios es a través de terciles. Para ello, a partir de la serie histórica de aportaciones del embalse se generan 3 intervalos con las aportaciones "por debajo de lo normal", "normales" y "por encima de lo normal". Por la forma de generar los terciles, la probabilidad climatológica de que la aportación de un invierno determinado se corresponda con un determinado tercil es del 33\%. La presentación por terciles es una forma habitual de suministrar la información de los pronósticos probabilistas estacionales. Clasificando los miembros del ensemble en los intervalos que corresponden a los terciles climatológicos, obtenemos que la probabilidad pronosticada de cada tercil será directamente proporcional a la población de miembros del ensemble que contiene.

En la Figura 6 se presenta la probabilidad de los terciles climatológicos (izquierda) y la de un ejemplo de producto de pronóstico que se genera en el módulo que se ha desarrollado (derecha).
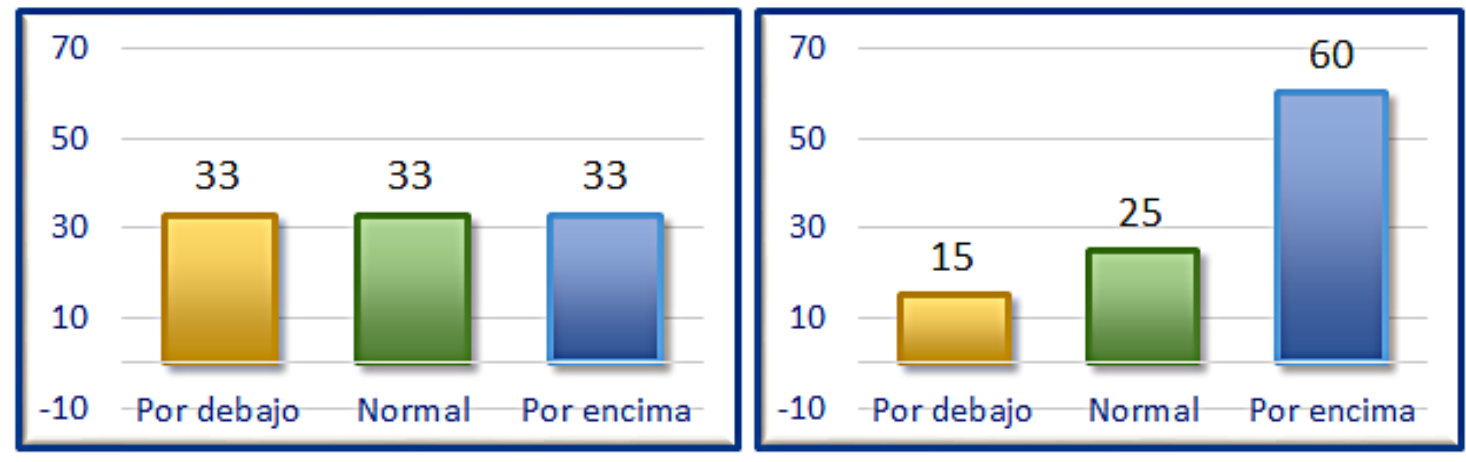

Figura 6. Ejemplo de representación en terciles

\section{Experimentación}

El sistema desarrollado se ha evaluado mediante la realización de una serie de experimentos en 4 embalses piloto sobre un periodo retrospectivo. Además de aportaciones al embalse, el sistema ha generado predicciones estacionales de precipitación en estaciones pluviométricas del entorno. El 
diseño de los experimentos afecta a la selección de los embalses y de las estaciones pluviométricas, los índices de variabilidad de la cobertura de nieve en otoño utilizados como predictores de la NAO invernal, y el periodo de cada año para el que se produce la predicción, el trimestre diciembre-enerofebrero, o un periodo ampliado que se extiende hasta el comienzo de la primavera y que fue requerido por los usuarios.

\section{Embalses seleccionados}

Los agentes implicados participantes en el proyecto de las Confederaciones Hidrográficas del Ebro, Duero y Tajo, así como de la Dirección General del Agua, han seleccionado los embalses piloto en los que se ha probado el funcionamiento del sistema desarrollado. Uno de los factores más relevantes para escogerlos ha sido la existencia de una correlación significativa entre la NAO y la precipitación en el trimestre DEF, que como se puede apreciar en la Figura 7 presenta una heterogénea distribución espacial en las distintas cuencas españolas. Además de este criterio, se intentó escoger embalses con series largas de observación completas cuyo estado estuviera relacionado muy directamente con la cantidad de precipitación acumulada en el trimestre. Por este motivo, se tuvo en cuenta la ubicación y el tamaño de la cuenca, la ausencia o presencia de regulación del caudal aguas arriba, las características del suelo de la cuenca, la aportación debida a la nieve y aguas subterráneas, las filtraciones, etc.

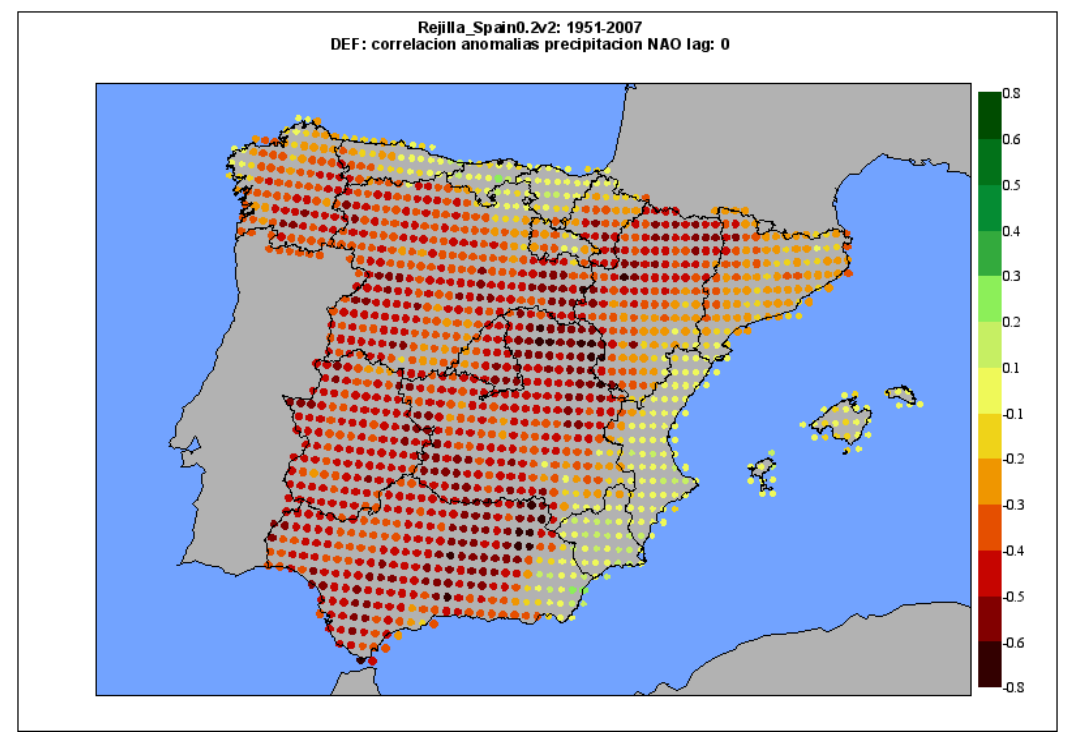

Figura 7. Correlación de la precipitación acumulada en diciembre-enero-febrero con el índice de la NAO promedio del mismo trimestre. Base de datos de precipitación utilizada: rejilla Spain02 (Herrera et al., 2012 )

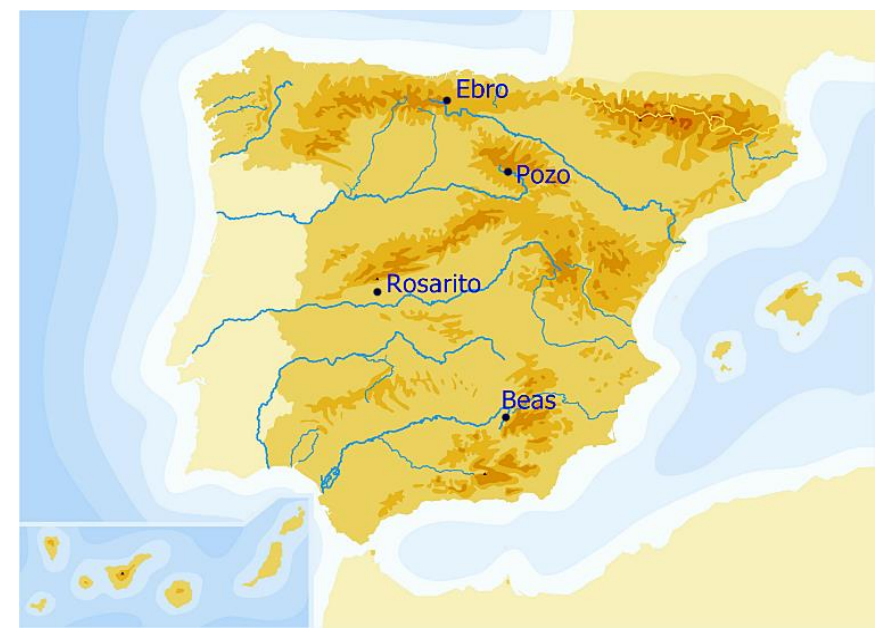

Figura 8. Embalses seleccionados 
Teniendo en cuenta estas consideraciones, los embalses piloto para los que se ha probado el sistema son: el embalse del Ebro (sugerido por la $\mathrm{CH}$ Ebro), el embalse de la Cuerda del Pozo (por la $\mathrm{CH}$ Duero), el de Rosarito (por la CH Tajo) y el del Tranco de Beas, cuya situación se muestra en la figura siguiente. El embalse del Tranco de Beas, en la $\mathrm{CH}$ del Guadalquivir, fue propuesto también por la alta correlación existente entre la precipitación invernal y la NAO.

Los datos de observaciones hidrológicos necesarios para estos experimentos han sido proporcionados por la Dirección General del Agua y son los existentes en el Sistema de Información del Anuario de Aforos (http://www.magrama.gob.es/es/agua/temas/evaluacion-de-los-recursos-hidricos/sistemainformacion-anuario-aforos/). Estas observaciones se utilizan en la segunda etapa del modelo de predicción conjuntamente con la serie de datos de la NAO en el periodo 1950 - 2012. Prácticamente no hay lagunas en estas series (ver Tabla 1).

\begin{tabular}{|l|l|l|l|}
\hline \multicolumn{2}{|c|}{ Variable: volumen de entrada } \\
\hline \multirow{2}{*}{ Indicativo } & \multirow{2}{*}{ Nombre del embalse } & \multicolumn{2}{|c|}{ No años con datos (1950 - 2012) } \\
\cline { 3 - 4 } & & NDEFM & DEF \\
\hline 2001_DGA & Cuerda del Pozo & 62 & 62 \\
\hline 3127_DGA & Rosarito & 53 & 53 \\
\hline 5001_DGA & Tranco de Beas & 62 & 62 \\
\hline 9801_DGA & Embalse del Ebro & 62 & 62 \\
\hline
\end{tabular}

Tabla 1. Información disponible de los embalses seleccionados

\section{Estaciones pluviométricas seleccionadas}

Para complementar la evaluación del sistema se han realizado también predicciones estacionales de precipitación. Esto ha permitido comprender mejor los resultados obtenidos. Para ello, se han seleccionado las estaciones pluviométricas en el entorno de los embalses que figuran en la Tabla 2.

Los datos pluviométricos de estas estaciones son los archivados en el Banco Nacional de Datos Climatológicos de la Agencia Estatal de Meteorología y cubren el periodo 1970 - 2014. Estas series presentan lagunas en algunas estaciones (ver Tabla 2).

\begin{tabular}{|c|c|c|c|}
\hline \multicolumn{4}{|c|}{ Variable: Precipitación } \\
\hline \multirow{2}{*}{ Indicativo } & \multirow{2}{*}{ Nombre de la estación } & \multicolumn{2}{|c|}{ № años con datos $(1970-2014)$} \\
\hline & & NDEFM & DEF \\
\hline \multicolumn{4}{|c|}{ Embalse Cuerda del Pozo } \\
\hline 2004_AEMET & Covaleda (a $12 \mathrm{~km}$ del embalse) & 38 & 38 \\
\hline 2011_AEMET & Cuerda del Pozo (en la orilla) & 36 & 37 \\
\hline \multicolumn{4}{|c|}{ Embalse Rosarito } \\
\hline 3422_AEMET & Pantano Rosarito (en la orilla) & 33 & 33 \\
\hline 3427E_AEMET & Pueblonuevo de Miramontes (a 8 km) & 34 & 35 \\
\hline \multicolumn{4}{|c|}{ Embalse Tranco de Beas } \\
\hline 7045_AEMET & Pontones (CH Segura. A $10 \mathrm{~km}$ ) & 35 & 39 \\
\hline 7056_AEMET & Santiago de la Espada (a $20 \mathrm{~km}$ ) & 38 & 38 \\
\hline \multicolumn{4}{|c|}{ Embalse del Ebro } \\
\hline 9008_AEMET & Arroyo de Valdearroyo (en la orilla) & 15 & 16 \\
\hline 90110_AEMET & $\begin{array}{l}\text { La Poblacion de Yuso (Iberduero. En la } \\
\text { orilla) }\end{array}$ & 21 & 23 \\
\hline
\end{tabular}

Tabla 2. Información disponible de las estaciones pluviométricas seleccionadas 


\section{Indices de la NAO y de variabilidad de la nieve}

La serie de índices de la NAO mensuales utilizada en el sistema que se ha desarrollado procede del Climate Prediction Center del National Weather Service de EEUU. El procedimiento de cálculo se describe

http://www.cpc.ncep.noaa.gov/products/precip/CWlink/daily ao index/history/method.shtml. Los valores de la NAO descargados del Climate Prediction Center cubren el periodo desde 1950 hasta la actualidad y no tienen lagunas.

Una parte importante del diseño de los experimentos realizados ha consistido en estudiar cuál es el índice de variabilidad de la cobertura de la nieve en el otoño con los que se trabaja en AEMET que mejor se comporta como predictor del sistema de predicción. En todos los experimentos, los datos de partida para calcular el índice de nieve proceden de productos de cobertura de nieve del National Snow and lce Data Center de EEUU.

Los experimentos que se han realizado han usado alternativamente los siguientes índices de variabilidad de la cobertura de nieve:

- SAl diario: índice de avance de la nieve en Eurasia diario. Se define como el coeficiente de regresión del ajuste lineal por mínimos cuadrados de la evolución diaria de la extensión de nieve en Eurasia (región geográfica comprendida entre $45^{\circ}-60^{\circ} \mathrm{N}, 0^{\circ}-180^{\circ} \mathrm{E}$ ) durante el mes de octubre. La extensión de nieve en Eurasia se ha calculado en AEMET utilizando los valores diarios en rejilla de la cobertura de nieve que proporciona el producto IMS Daily Northern Hemisphere Snow and Ice Analysis. Este dataset procedente del Interactive Multisensor Snow and Ice Mapping System (IMS) del National Ice Center de EEUU, en el momento de calcular el índice, disponía de datos desde 1999. El producto está derivado a partir de diferentes fuentes de datos, tanto satelitales como in-situ.

- SAl semanal: índice de avance de la nieve en Eurasia semanal. Se define igual que el SAI diario pero los datos de partida de cobertura de nieve en rejilla tienen frecuencia semanal y se usan los correspondientes a las semanas 40 a la 44. La cobertura de nieve en rejilla utilizada para el cálculo de la extensión de nieve en Eurasia a escala semanal es la del producto Northern Hemisphere EASE-Grid Weekly Snow Cover http://nsidc.org/data/nsidc-0046), obtenido a partir del producto de la NOAA de Snow Cover Extent (SCE) y posteriormente remapeado a la rejilla EASE. El producto SCE fue derivado mediante la interpretación manual de los datos de satélite de distintos instrumentos (AVHRR, GOES, etc.) Así se pueden usar datos anteriores a 1997, obteniendo una serie notablemente más larga, (1973 - 2013). Esta degradación espacial y temporal tiene su coste ya que la correlación SAI - NAO(DEF) es más alta utilizando el SAI diario que el semanal.

- SVI diario: índice de variabilidad diaria de la nieve en Norteamérica. Este índice, que se calcula en AEMET a partir del mismo producto de cobertura de nieve a escala diaria procedente del National Snow and Ice Data Center de EEUU que el SAI diario, mide el promedio de la variabilidad absoluta diaria de la extensión de la nieve entre las semanas 40 y 44

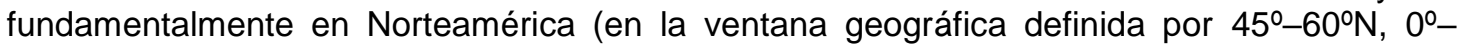
180W). El primer año de la serie disponible en el momento de calcular el índice es 1999.

\section{Experimentos realizados con diferentes índices de variabilidad de la nieve}

Para evaluar el comportamiento del sistema se han llevado a cabo una serie de hindcast con diferentes predictores. Para cada año del periodo elegido, el sistema hace el pronóstico sin tener en cuenta en el proceso el año en cuestión.

Dado que el proceso de evaluación es complejo e involucra varios parámetros, se ha nombrado como un sistema diferente a cada hindcast en función del predictor y del periodo de la serie del predictor utilizado. La implementación del sistema es la misma, la diferencia entre los experimentos está en los datos utilizados para hacer la regresión lineal. Aunque en el momento de diseñar los experimentos la serie de datos de precipitación estaba disponible hasta 2014 , la de aportaciones a los embalses termina en 2011. En la Tabla 3 se muestra una descripción detallada de los mismos. 
DESCRIPCIÓN DE LOS EXPERIMENTOS

\begin{tabular}{|c|c|c|c|c|}
\hline EXP & $\begin{array}{l}\text { PREDICTOR DE } \\
\text { LA NAO (DEF) }\end{array}$ & $\begin{array}{c}\text { PERIODO } \\
\text { BASE PARA } \\
\text { OBTENER EL } \\
\text { COEFICIENTE } \\
\text { DE } \\
\text { REGRESION * }\end{array}$ & $\begin{array}{c}\text { PERIODO } \\
\text { BASE PARA } \\
\text { ENCONTRAR } \\
\text { LAS } \\
\text { SITUACIONES } \\
\text { HISTÓRICAS } \\
\text { SIMILARES }{ }^{* \star}\end{array}$ & $\begin{array}{l}\text { PERIODO } \\
\text { DEL } \\
\text { HINDCAST }\end{array}$ \\
\hline SVI_NAmerica_daily & $\begin{array}{l}\text { Snow Variability Index } \\
\text { en América del } \\
\text { Norte. Los productos } \\
\text { de cobertura nivosa } \\
\text { de partida tienen una } \\
\text { frecuencia diaria } \\
\text { (daily). }\end{array}$ & $1999-2014$ & $\begin{array}{l}\text { 1950-2011 } \\
\text { (aportaciones) } \\
1970-2014 \text { (pcp) }\end{array}$ & $\begin{array}{l}\text { 1999-2011 } \\
\text { (aportaciones) } \\
1999-2014 \text { (pсp) }\end{array}$ \\
\hline SAI_Eurasia_daily & $\begin{array}{lr}\text { Snow Advance Index } \\
\text { en Eurasia. Los } \\
\text { productos } \\
\text { cobertura nivosa de } \\
\text { partida tienen una } \\
\text { frecuencia diaria } \\
\text { (daily). }\end{array}$ & $1999-2014$ & $\begin{array}{l}\text { 1950-2011 } \\
\text { (aportaciones) } \\
1970-2014 \text { (pcp) }\end{array}$ & $\begin{array}{l}\text { 1999-2011 } \\
\text { (aportaciones) } \\
1999-2014 \text { (pсp) }\end{array}$ \\
\hline SAI_Eurasia_weekly & $\begin{array}{l}\text { Snow Advance Index } \\
\text { en Eurasia. Los } \\
\text { productos } \\
\text { cobertura nivosa de } \\
\text { partida tienen una } \\
\text { frecuencia semanal } \\
\text { (weekly). }\end{array}$ & $1999-2013$ & $\begin{array}{l}\text { 1950-2011 } \\
\text { (aportaciones) } \\
1970-2014 \text { (pcp) }\end{array}$ & $\begin{array}{l}\text { 1999-2011 } \\
\text { (aportaciones) } \\
1999-2013 \text { (pсp) }\end{array}$ \\
\hline SAI_Eurasia_weekly_ext & $\begin{array}{l}\text { Snow Advance Index } \\
\text { en Eurasia. Los } \\
\text { productos } \\
\text { cobertura nivosa de } \\
\text { partida tienen una } \\
\text { frecuencia semanal } \\
\text { (weekly) sobre un } \\
\text { periodo extendido. }\end{array}$ & $1973-2013$ & $\begin{array}{l}\text { 1950-2011 } \\
\text { (aportaciones) } \\
1970-2014 \text { (pcp) }\end{array}$ & $\begin{array}{l}\text { 1973-2011 } \\
\text { (aportaciones) } \\
1973-2013 \text { (pсp) }\end{array}$ \\
\hline
\end{tabular}

* Excluyendo el año que se predice.

Tabla 3. Descripción de los experimentos.

** Todos los años del periodo con datos disponibles de aportaciones/precipitación, excluyendo los del año que se predice.

\section{Periodo de acumulación de aportaciones y precipitación para el que se generan predicciones}

Ante el interés mostrado por los gestores del agua de disponer de las previsiones de aportaciones totales en un periodo más largo que el trimestre DEF, los experimentos se han repetido usando como periodo de pronóstico noviembre-diciembre-enero-febrero-marzo (NDEFM). Esta extensión del periodo se puede justificar por ser los meses del trimestre invernal (DEF) aquellos en los que se registra normalmente mayor cantidad de precipitación, y por la fusión de parte de la nieve caída en el trimestre invernal durante la primavera. También existen estudios que indican la existencia de una relación estadística entre la NAO del trimestre invernal y la circulación atmosférica en los meses de primavera siguientes (Hori and Yasunari, 2003; Castro et al., 2011; Ogi et al. 2003) que apoyan esta aproximación. 
Cuando se amplía el periodo de acumulación de aportaciones y precipitación a NDEFM, en el primer paso de la predicción se sigue utilizando la correlación entre el índice de variabilidad de la nieve en otoño y la $N A O(D E F)$, y en el segundo la influencia de la $N A O(D E F)$ en las aportaciones/precipitaciones acumuladas históricamente.

\section{Evaluación del sistema de predicción estacional}

\section{Métricas utilizadas para la verificación}

La calidad de las predicciones del volumen de entrada y de la precipitación obtenidas en los distintos experimentos descritos anteriormente, ha sido evaluada mediante una verificación objetiva desde el punto de vista determinista y probabilístico.

La predicción determinista de los sistemas de predicción (la media del ensemble) se ha verificado calculando el coeficiente de correlación ( $r$ ) entre los valores de las anomalías de observaciones y predicciones, para cada uno de los puntos de observación, para las dos variables (volumen de entrada y precipitación) y periodos de acumulación - DEF y NDEFM- .

Desde el punto de vista probabilístico, se han calculado, para las mismas variables, periodos y puntos de observación, el Relative Operating Characteristic área (ROC área) y el Brier Skill Score (BSS), para dos eventos, (valores por encima/debajo del tercil superior/inferior).

El Relative Operating Characteristic área (ROC área) es un índice de verificación, que se calcula a partir de la denominada curva ROC (Figura 9).

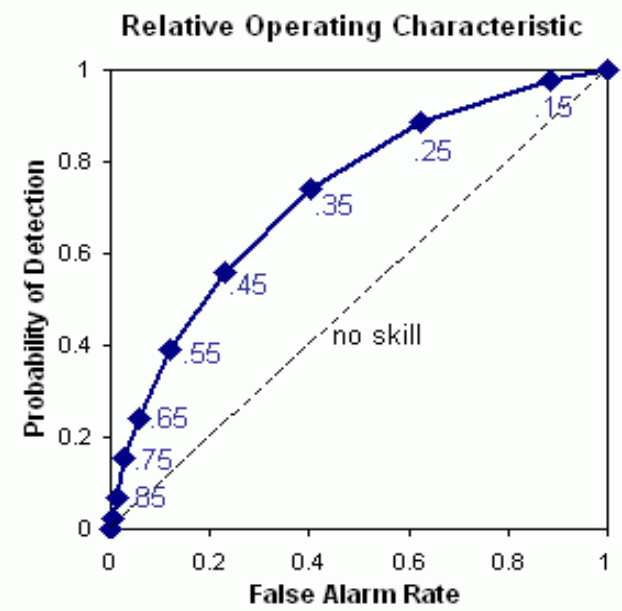

Figura 9. Curva ROC

Esta curva mide el grado de discriminación de un sistema de predicción. La discriminación es la capacidad de distinguir un resultado categórico de otro, incluso si las probabilidades de la predicción tienen sesgos o problemas de calibración. La curva ROC se calcula para cada categoría de predicción individualmente. Si, por ejemplo, la categoría en estudio es "valores por encima de lo normal", el área ROC indicaría la probabilidad de discriminar con éxito las observaciones con valores superiores a lo normal de aquellas cuyos valores no son superiores a lo normal.

Para el cálculo del área ROC las predicciones se clasifican en $n$ intervalos decrecientes de probabilidad prevista de la categoría en estudio. La muestra de predicciones en intervalos subsiguientes es cada vez mayor, ya que contiene las predicciones de los intervalos anteriores. En cada uno de los $n$ intervalos se obtiene cuál ha sido el número de aciertos frente al total de observados de esa categoría, y de forma similar se obtiene la proporción de falsas alarmas. Con estos valores de la tasa de aciertos y de falsas alarmas para un umbral de probabilidad concreto se construye cada uno de los puntos de la curva ROC. El área ROC es la delimitada por la curva ROC y el eje de abcisas. Toma valores entre 0 y 1 . El valor 
0.5 (curva ROC coincidente con la diagonal de la gráfica) corresponde a un sistema de predicción probabilístico que no tiene pericia, 1 indica una discriminación perfecta, y 0 indica una perfectamente mala discriminación.

Es importante resaltar que las curvas ROC sólo miden la habilidad para discriminar entre dos posibles resultados, pero no dicen nada sobre la fiabilidad, ni son sensibles al sesgo. Errores sistemáticos de localización estarían presentes, por ejemplo, si las predicciones de "valores por encima de lo normal" tienen probabilidades que son inferiores a su tasa observada de ocurrencia, incluso si hubiera alguna discriminación para esta categoría. Los errores sistemáticos del nivel de confianza estarían presentes, por ejemplo, cuando las predicciones indican una mayor confianza (es decir, muestran probabilidades alejadas de 0.333) que la que es justificada por el grado de discriminación.

El Brier Score (BS) es la versión probabilista del error cuadrático medio (MSE). Mide la suma de las diferencias al cuadrado en el espacio de las probabilidades para una predicción probabilística en el caso de eventos binarios (a la observación de una categoría se le asigna un valor de probabilidad observada 1 , y a la no ocurrencia de la categoría se le asigna un valor de probabilidad observada 0 ). El valor del BS oscila entre 0 y 1 , siendo 0 el valor que tendría una predicción perfecta. El BS indica hasta qué punto la predicción no tiene éxito en la discriminación de una categoría observada específica, y/o tiene sesgos sistemáticos de localización y del nivel de confianza en la probabilidad de ocurrencia de esa categoría. Por tanto, el BS refleja el grado de ausencia de discriminación, fiabilidad y/o resolución.

Para poder comparar el sistema de predicción en estudio con otro sistema de referencia se usa el Brier Skill Score (BSS), índice derivado del BS que se define como:

$$
\mathrm{BSS}=1-\mathrm{BS} / \mathrm{BS} \text { ref }
$$

El BSS toma valores entre $-\infty$ y 1 . Valores positivos del BSS indican más pericia que el sistema de referencia, generalmente la climatología. Valores negativos o cero indican que el sistema de predicción no la tiene. A diferencia del ROC área, y por estar basado en el BS de la predicción y de la climatología, el BSS tiene en cuenta errores de discriminación y/o sesgos de localización y de nivel de confianza.

Una definición completa de todos estos índices se puede encontrar en Wilks (2006).

\section{Datos utilizados para la verificación}

Dependiendo del embalse/estación pluviométrica, las series de años utilizadas para realizar la verificación de las predicciones son diferentes. En las tablas 4 y 5 se describen el número de años usados en la verificación en función de la variable (volumen de entrada y precipitación) y experimento.

\begin{tabular}{|c|c|c|c|c|c|}
\hline \multicolumn{6}{|c|}{ Variable: Volumen de Entrada } \\
\hline \multirow{3}{*}{ Indicativo } & \multirow{3}{*}{ Nombre del embalse } & \multicolumn{4}{|c|}{ № años con datos } \\
\hline & & \multicolumn{2}{|c|}{$1973-2013$ * } & \multicolumn{2}{|c|}{$1999-2013^{\text {** }}$} \\
\hline & & NDEFM & DEF & NDEFM & DEF \\
\hline 2001_DGA & Cuerda del Pozo & 39 & 39 & 13 & 13 \\
\hline 3127_DGA & Rosarito & 38 & 38 & 13 & 13 \\
\hline 5001_DGA & Tranco de Beas & 39 & 39 & 13 & 13 \\
\hline 9801_DGA & Embalse del Ebro & 39 & 39 & 12 & 13 \\
\hline
\end{tabular}

Tabla 4. Número de años utilizados para la verificación de la variable Volumen de Entrada

* Serie de años seleccionada para el experimento "SAI_Eurasia_weekly_ext".".

** Serie de años seleccionada para los experimentos "SVI_NAmerica_daily", "SAI_Eurasia_daily" y "SAI_Eurasia_weekly".

Variable: Precipitación

\begin{tabular}{|l|l|l|l|}
\hline \multicolumn{2}{|l|}{ Variable: Precipitación } & \multicolumn{2}{|c|}{ № años con datos } \\
\cline { 3 - 4 } & Nombre de la estación & $1973-2013^{\text {* }}$ & $1999-2013^{\text {** }}$ \\
\hline
\end{tabular}




\begin{tabular}{|c|c|c|c|c|c|}
\hline & & NDEFM & DEF & NDEFM & DEF \\
\hline \multicolumn{6}{|c|}{ Embalse Cuerda del Pozo: } \\
\hline 2004_AEMET & Covaleda (Situada a $12 \mathrm{Km}$ ) & 38 & 38 & 13 & 13 \\
\hline 2011_AEMET & $\begin{array}{l}\text { Cuerda del Pozo (En la orilla del } \\
\text { embalse) }\end{array}$ & 36 & 37 & 10 & 11 \\
\hline \multicolumn{6}{|c|}{ Embalse Rosarito: } \\
\hline 3422_AEMET & $\begin{array}{l}\text { Pantano Rosarito (En la orilla del } \\
\text { embalse) }\end{array}$ & 33 & 33 & 11 & 11 \\
\hline 3427E_AEMET & $\begin{array}{l}\text { Pueblonuevo de Miramontes } \\
\text { (Situada a } 8 \mathrm{Km} \text { ) }\end{array}$ & 34 & 35 & 15 & 15 \\
\hline \multicolumn{6}{|c|}{ Embalse Tranco del Beas: } \\
\hline 7045_AEMET & $\begin{array}{l}\text { Pontones (CH Segura. Situada a } \\
10 \mathrm{Km} \text { del embalse) }\end{array}$ & 35 & 39 & 11 & 14 \\
\hline 7056_AEMET & $\begin{array}{l}\text { Santiago de la Espada (Situada a } \\
20 \mathrm{Km} \text { ) }\end{array}$ & 38 & 38 & 12 & 12 \\
\hline \multicolumn{6}{|c|}{ Embalse del Ebro: } \\
\hline 9008_AEMET & $\begin{array}{l}\text { Arroyo de Valdearroyo (Situada en } \\
\text { la orilla del embalse) }\end{array}$ & 15 & 16 & 10 & 11 \\
\hline 90110_AEMET & $\begin{array}{l}\text { La Población de Yuso (Iberduero. } \\
\text { En una de las orillas del embalse) }\end{array}$ & 21 & 23 & 6 & 6 \\
\hline
\end{tabular}

Tabla 5. Número de años utilizados para la verificación de la variable Precipitación

* Serie de años seleccionada para el experimento "SAI_Eurasia_weekly_ext".".

** Serie de años seleccionada para los experimentos "SVI_NAmerica_daily", "SAI_Eurasia_daily" y "SAl_Eurasia_weekly".

La verificación se ha realizado utilizando las salidas directas de los sistemas de predicción, sin calcular anomalías, ni corregir el bias que pudiera existir. Para la clasificación por categorías, tanto en las observaciones como en el hindcast, se utilizan los mismos terciles, que han sido obtenidos a partir de la serie histórica de observaciones de la que se dispone de cada embalse (desde 1950) o estación climatológica (desde 1970). En el sistema de referencia utilizado para el cálculo del índice BSS (climatología), cada categoría calculada con estos terciles tiene asignado un 33'33\% de probabilidad.

\section{Resultados}

\section{Verificación de las predicciones para el periodo DEF:}

La Figura 10 muestra los valores obtenidos en la verificación, desde un punto de vista determinista, de los experimentos realizados con los cuatro sistemas de predicción descritos anteriormente, en cada uno de los puntos de observación en el periodo de hindcast 1999 hasta 2013. Para ello, consideramos como valor previsto la media de todos los valores obtenidos por los 1500 miembros de nuestro conjunto de predicciones.

Si nos centramos en las aportaciones, vemos que presenta valores de correlación significativos (mayores que 0.5), incluso superiores a 0.7 en algunos casos, para todos los embalses en los dos sistemas de predicción que utilizan índices de variabilidad de la nieve (en Eurasia y en Norteamérica) basados en datos de cobertura de nieve en rejilla de frecuencia diaria para predecir la NAO(DEF). Sin embargo, la correlación es claramente inferior (en torno al 0.3) para los sistemas que representan la variabilidad de la nieve en Eurasia utilizando productos de cobertura de nieve de frecuencia semanal, incluso aunque se utilice una serie histórica más larga de datos en la primera etapa de predicción de la NAO invernal (sistema “SAI_Eurasia_weekly_ext').

En el caso de la variable precipitación, los valores obtenidos por los sistemas basados en productos diarios de cobertura de nieve, excepto para las estaciones próximas al Embalse del Ebro, son también bastante elevados en general, destacando el sistema basado en el índice de variabilidad de la nieve en Norteamérica, con valores en torno a 0.8. Al igual que para las aportaciones, para los sistemas que 
utilizan como predictor de la NAO el índice de variabilidad de la nieve en Eurasia basado en datos de frecuencia semanal, los valores son inferiores a 0.5 prácticamente en todos los casos.



Figura 10. Coeficiente de correlación para el periodo DEF por embalses, entre observaciones y la media del ensemble de cada uno de los cuatro modelos de predicción, tomando como periodo de verificación todos los años con datos desde 1999 a 2013. Los valores mostrados en forma de barras de colores en función del sistema de predicción, corresponden a los obtenidos para las aportaciones a cada embalse. Los valores obtenidos por cada sistema de predicción para la precipitación en las diferentes estaciones pluviométricas se muestran con puntos en forma de círculos y triángulos en la vertical de la aportación correspondiente.

Los valores de correlación para las predicciones de precipitación en las estaciones cercanas al embalse del Ebro son las que más difieren de los de las predicciones de las aportaciones, y son valores bajos, e incluso negativos en algún caso. Hay que tener en cuenta que, sobre todo en el embalse del Ebro, la serie de datos de precipitación es más corta que la de las aportaciones, y presenta muchas lagunas. Esto tiene un impacto tanto en el segundo paso del sistema de predicción, como en la propia verificación (ver tablas 1, 2, 4 y 5).

Desde un punto de vista probabilista, los resultados de los índices BSS y áreas ROC para el tercil superior e inferior se muestran en las Figuras 11 y 12.

Al igual que cuando se mide la pericia con el coeficiente de correlación, se observan diferencias significativas entre los sistemas generados a partir del producto de cobertura de nieve diario y los basados en el producto semanal. Se obtienen mejores resultados con los sistemas basados en el diario, al igual que ocurría con los índices deterministas.

Si nos fijamos en la verificación de las predicciones de las aportaciones a los embalses, vemos que, salvo en el caso del Embalse del Ebro, los valores del BSS -tanto para el tercil superior como para el inferior- son positivos (excepto un caso aislado) para todos los sistemas de predicción, lo cual implica que todos tienen más pericia que la climatología usada como modelo de referencia. Incluso se alcanzan valores de BSS superiores a 0.3 en algunos embalses para los sistemas basados en el producto de cobertura de nieve diario. Los valores de BSS para la precipitación son, en general, algo más bajos, pero como se ha mencionado anteriormente, las series pluviométricas utilizadas en el segundo paso de predicción son mucho más cortas que las de aportaciones, y además el periodo de verificación no coincide tampoco exactamente con el de las aportaciones. 
Respecto al área ROC, excepto en el caso del Embalse del Ebro para el tercil inferior, cabe destacar que no sólo se alcanzan valores superiores a 0.5 , sino que en muchos casos están en torno a 0.8 o son incluso superiores a 0.9 , lo cual implica que estos sistemas presentan una clara pericia para discriminar los terciles superior e inferior. Normalmente, los mejores resultados se obtienen en el caso del tercil superior.



Figura 11. BSS para valores de aportaciones/precipitación por encima y por debajo de los valores normales, para el periodo $\mathrm{DEF}$, por embalses y sistema de predicción, tomando como periodo de verificación todos los años con datos desde 1999 a 2013. Los valores mostrados en forma de barras de colores en función del sistema de predicción, corresponden a las aportaciones a cada embalse. Los valores de este índice de verificación obtenidos por cada sistema de predicción para la precipitación en las diferentes estaciones pluviométricas se muestran con puntos en forma de círculos y triángulos en la vertical del de la aportación correspondiente.

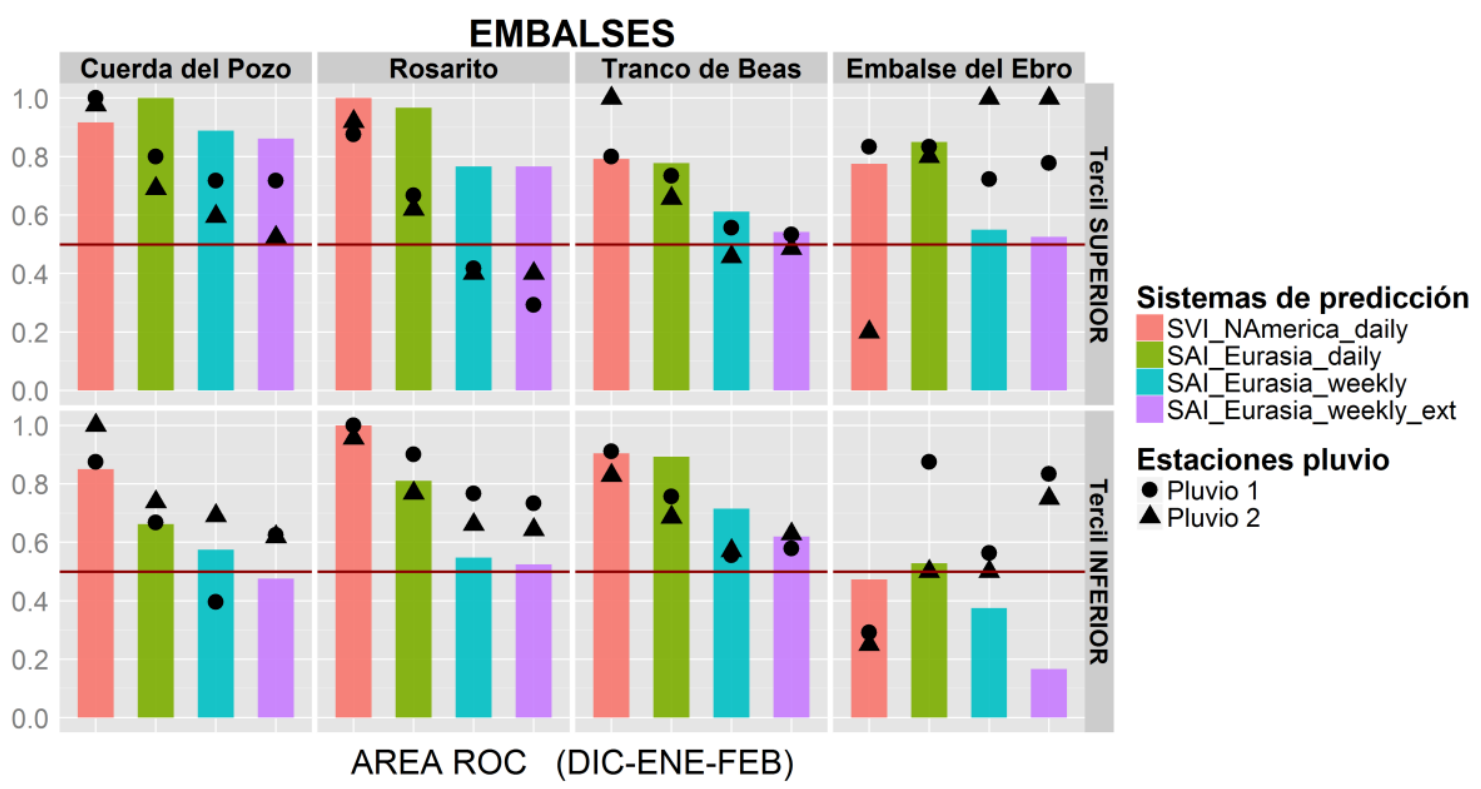

Figura 12. Área ROC para valores por encima y por debajo de los valores normales, para el periodo DEF, por embalses y sistema de predicción, tomando como periodo de verificación todos los años con datos desde 1999 a 2013. Los valores mostrados en forma de barras de colores en función del sistema de predicción, corresponden a los valores obtenidos para las aportaciones a cada embalse. Los valores de este índice de verificación obtenidos por cada sistema de predicción para la precipitación en las diferentes estaciones pluviométricas se muestran con puntos en forma de círculos y triángulos en la vertical de la aportación correspondiente.

Los valores de área ROC de las predicciones de precipitación indican en su mayoría un comportamiento muy similar al de las aportaciones, obteniendo valores más altos los sistemas de predicción basados 
en el producto de cobertura de nieve de frecuencia diaria. En general, en el caso del tercil superior, la pericia de las predicciones de las aportaciones es mayor que la de las precipitaciones. En el caso del tercil inferior, es más corriente encontrar que se discrimina mejor esta categoría en las predicciones de precipitación que en las de aportaciones. El hecho de que las series de precipitación sean más cortas y recientes que las de las aportaciones podría ser un factor que explicara este comportamiento (por la existencia de periodos húmedos que no están siendo muestreados por el modelo en el caso de la precipitación), así como el carácter más local de las observaciones pluviométricas, o que el modelo utilizado sea muy sencillo y no tenga en cuenta otros factores que puedan ser importantes para las aportaciones en periodos más secos.

Las predicciones de precipitación presentan algunas particularidades en el caso del entorno del Embalse del Ebro. En principio se podría esperar un peor comportamiento de cualquiera de los sistemas de predicción en esta área geográfica, por el hecho de que en la zona norte de España los valores de correlación entre el índice de la NAO y la precipitación son más bajos que en el resto de los embalses analizados en otras cuencas. Sin embargo, se observa que el área ROC de la precipitación es normalmente superior al de las aportaciones. El sistema de predicción que utiliza una serie histórica más larga (que tiene una mayor memoria de la variabilidad de la NAO, aunque utilice un peor indicador de la evolución de la cobertura de nieve) discrimina especialmente bien la precipitación, pero no las aportaciones en este embalse. Aparte de que no coincidan completamente los periodos de verificación de las dos variables, y que las series pluviométricas sean más cortas, hay que tener en cuenta que las aportaciones se ven afectadas por otros factores que pueden tener más peso en este embalse y que no han sido tenidos en cuenta en el sencillo método que se ha adoptado, como el retardo en la escorrentía producida por la fusión de la nieve, los diferentes tipos de suelo, las aguas subterráneas, el control de las corrientes de los ríos, o el estado de humedad del suelo a comienzos del invierno. Finalmente señalar que, aunque algunos sistemas de predicción son capaces de discriminar el tercil húmedo y el seco en el caso de la precipitación, el índice BSS es muy bajo. Ello podría indicar problemas de sesgos en la predicción.

Es de destacar que, en general, los valores de los índices de verificación obtenidos por los sistemas "SAl_Eurasia_weekly” y "SAI_Eurasia_weekly_ext" son muy parecidos. La única diferencia entre ambos es la longitud de la serie de datos del índice de variabilidad de la nieve y de la NAO en el primer paso de predicción. En los embalses de las cuencas atlánticas analizados, para este primer paso de predicción de la NAO, no parece necesario disponer de un registro muy largo del índice de variabilidad de la nieve en los sistemas basados en los productos de cobertura de nieve de frecuencia semanal.

Por último, y con objeto de analizar la influencia de la elección del periodo de verificación en los resultados obtenidos, se ha verificado el sistema "SAl_Eurasia_weekly_ext" en dos periodos distintos: 1999-2013 (cuyos resultados son los presentados hasta ahora), y 1973-2013, obteniéndose mejores resultados para el periodo de verificación más largo (ver Anexo I).

\section{Verificación de las predicciones para el periodo de acumulación extendido a los meses NDEFM}

La figura 12 muestra los coeficientes de correlación obtenidos en la verificación desde un punto de vista determinista, de los cuatro sistemas de predicción, tomando como periodo de verificación todos los años con datos desde 1999 hasta 2013. Para ello, consideramos como valor previsto la media de todos los valores obtenidos por los 1500 miembros de nuestro conjunto de predicciones.

Los coeficientes de correlación para las aportaciones presentan valores similares a los obtenidos para el trimestre DEF, o ligeramente inferiores en algunos casos. Para la variable precipitación son, en general, ligeramente superiores. Sigue existiendo, por tanto, una correlación significativa a excepción del Embalse del Ebro.

Las figuras 13 y 14 muestran los índices BSS y área ROC para el tercil superior e inferior para las predicciones probabilísticas de aportaciones y precipitación. Los resultados siguen mostrando la diferente pericia existente entre los sistemas generados a partir de los predictores que utilizan productos de cobertura de nieve diarios y los basados en productos de frecuencia semanal.

Si nos fijamos en el índice BSS, se aprecia un comportamiento muy similar en las aportaciones al obtenido para el trimestre DEF, salvo en el caso del Embalse del Ebro. En este embalse, se encuentra 
que para el tercil superior el extender el periodo de acumulación a NDEFM produce una mejora significativa, pasando de tener valores de BSS negativos en varios sistemas de predicción a tener valores positivos, lo cual implica que la predicción de aportaciones presenta más habilidad que la climatología en el periodo extendido NDEFM.

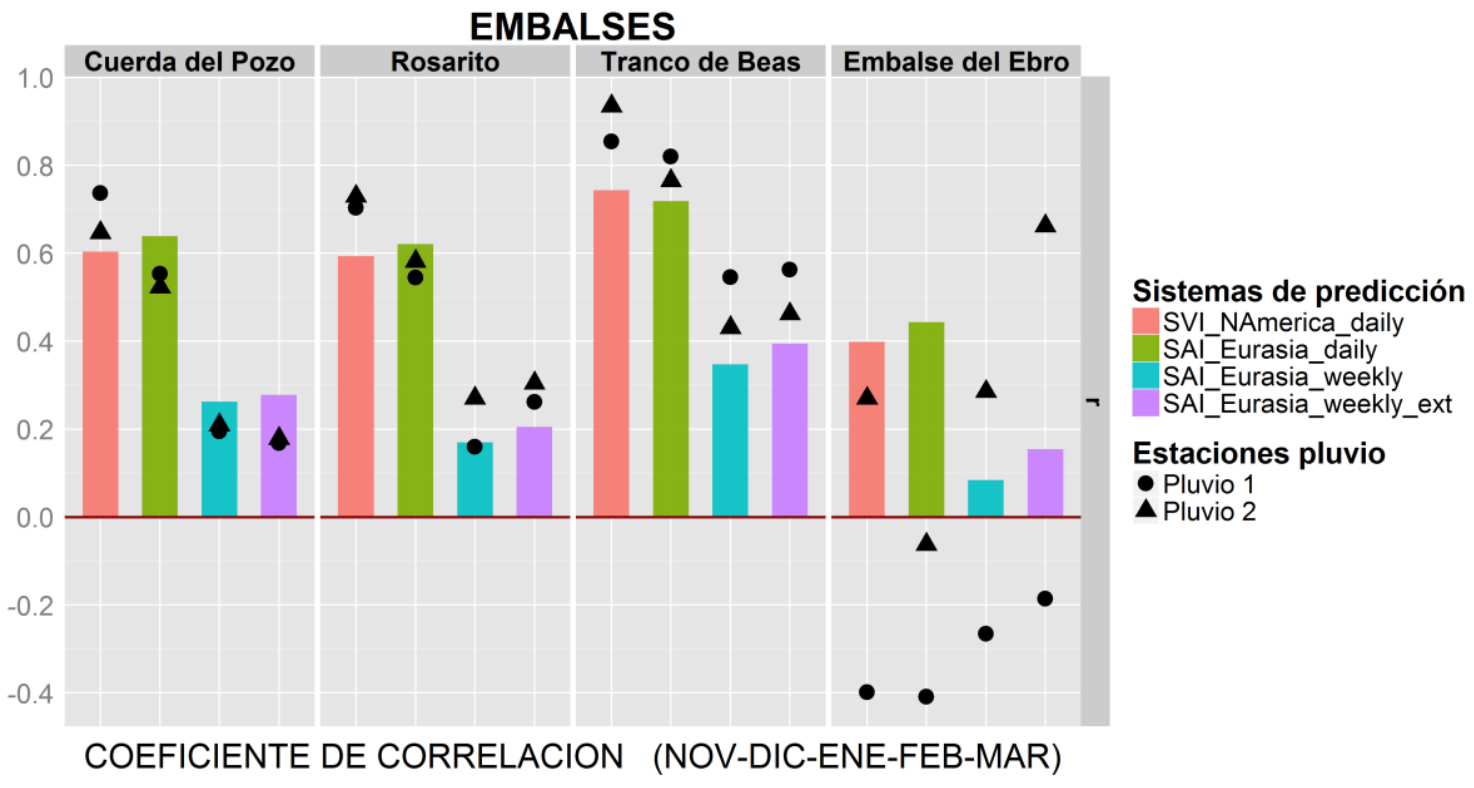

Figura 12. Coeficiente de correlación para el periodo NDEFM por embalses, entre observaciones y la media del ensemble de cada uno de los cuatro modelos de predicción, tomando como periodo de verificación todos los años con datos desde 1999 a 2013. Los valores mostrados en forma de barras de colores en función del sistema de predicción, corresponden a los obtenidos para las aportaciones a cada embalse. Los valores obtenidos por cada sistema de predicción para la precipitación en las diferentes estaciones pluviométricas se muestran con puntos en forma de círculos y triángulos en la vertical de la aportación correspondiente.

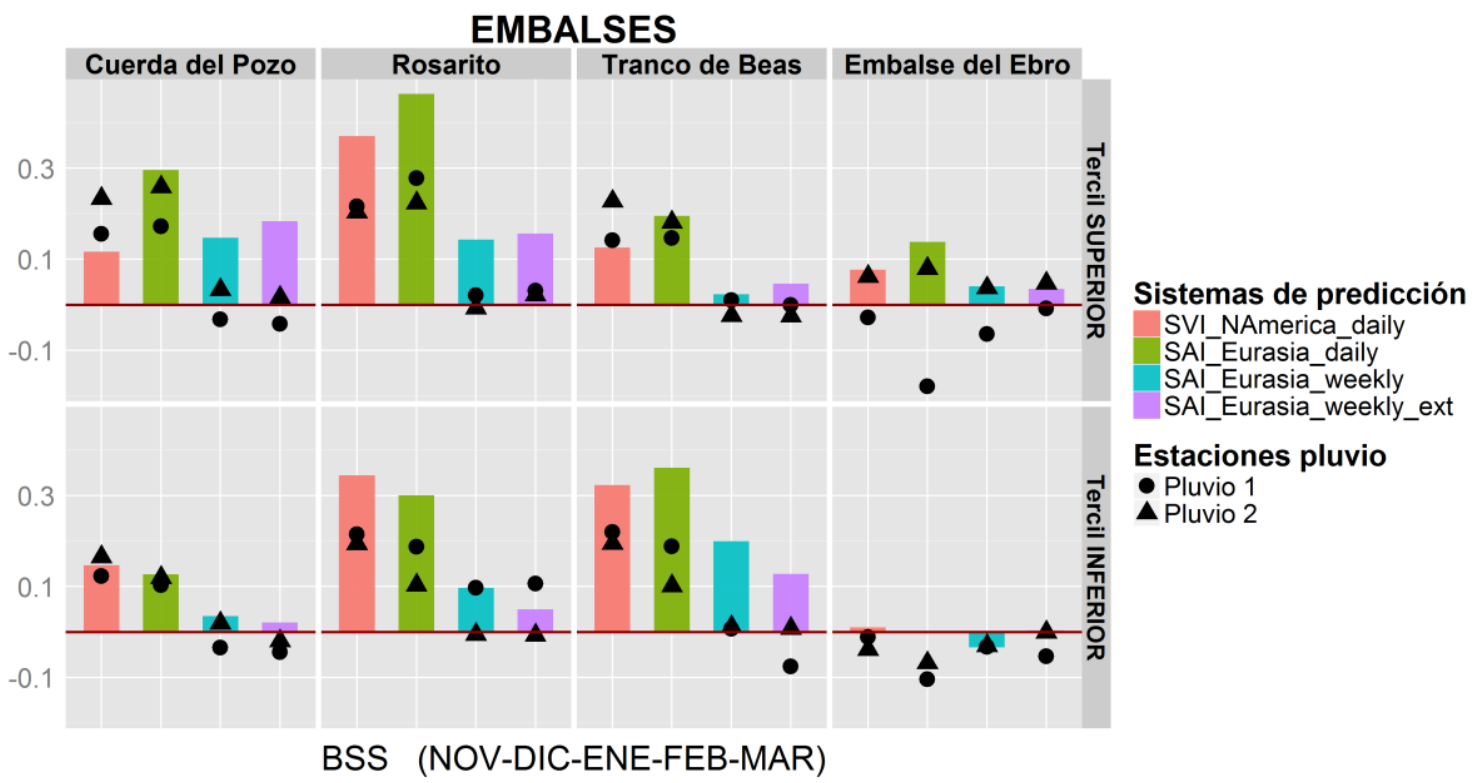

Figura 13. BSS para valores de aportaciones/precipitación por encima y por debajo de los valores normales, para el periodo NDEFM, por embalses y sistema de predicción, tomando como periodo de verificación todos los años con datos desde 1999 a 2013. Los valores mostrados en forma de barras de colores en función del sistema de predicción, corresponden a las aportaciones a cada embalse. Los valores de este índice de verificación obtenidos por cada sistema de predicción para la precipitación en las diferentes estaciones pluviométricas se muestran con puntos en forma de círculos y triángulos en la vertical del de la aportación correspondiente.

Respecto al área ROC, excepto en el Embalse del Ebro, los valores obtenidos no presentan, en general grandes diferencias con respecto a los correspondientes al trimestre DEF. Se observa, no obstante, 
una tendencia a valores ligeramente inferiores para el tercil húmedo y ligeramente superiores para el caso del tercil seco. Es decir, la pericia para discriminar periodos húmedos y secos tiende a igualarse. Otro resultado a destacar es la mejora de la discriminación del tercil inferior en las aportaciones (no así en la precipitación) para el Embalse del Ebro, obteniéndose para el periodo NDEFM valores mayores que 0.5 en los sistemas de predicción basados en los productos diarios de variabilidad de la nieve (incluso 0.75 para uno de ellos) y entre 0.4 y 0.5 para los otros dos sistemas. Una posible explicación para ello podría ser el que ampliar el periodo de acumulación supone tener en cuenta indirectamente la contribución al volumen de entrada al embalse procedente de la fusión de la nieve caída en el trimestre DEF, y esta fuente de las aportaciones puede ser muy importante en el caso del Embalse del Ebro.



Figura 14. Área ROC para valores por encima y por debajo de los valores normales, para el periodo NDEFM, por embalses y sistema de predicción, tomando como periodo de verificación todos los años con datos desde 1999 a 2013. Los valores mostrados en forma de barras de colores en función del sistema de predicción, corresponden a los valores obtenidos para las aportaciones a cada embalse. Los valores de este índice de verificación obtenidos por cada sistema de predicción para la precipitación en las diferentes estaciones pluviométricas se muestran con puntos en forma de círculos y triángulos en la vertical de la aportación correspondiente.

\section{Conclusiones}

Se ha desarrollado un método empírico de predicción estacional en dos pasos que produce predicciones probabilísticas de aportaciones a los embalses o de precipitación acumulados en el periodo invernal, y que utiliza como base series temporales de datos del índice de la NAO, de índices de variabilidad de la nieve en otoño, de aportaciones a los embalses y de precipitación. El método desarrollado se ha evaluado realizando diferentes experimentos sobre un periodo de hindcast (19992013) en 4 embalses piloto en las cuencas del Ebro, Duero, Tajo y Guadalquivir. Las principales conclusiones de la verificación objetiva de las predicciones producidas por los experimentos llevados a cabo son las siguientes:

- La pericia del sistema de predicción depende del embalse. Hay una clara diferencia de comportamiento entre los embalses piloto de las cuencas del Guadalquivir, Tajo y Duero, y el situado en la cabecera del Ebro.

- Las predicciones estacionales de las aportaciones y precipitación en los embalses piloto seleccionados en las cuencas del Guadalquivir, Duero y Tajo muestran una pericia significativa para discriminar tanto el carácter húmedo como el carácter seco del próximo trimestre invernal (DEF). Aunque los periodos de verificación no coinciden completamente, en estos embalses, las predicciones de aportaciones obtienen índices de verificación ligeramente mejores/peores que las de precipitación para el tercil superior/inferior. En la mayoría de los casos, las predicciones producidas mejoran a la climatología, y las medias del conjunto de predicciones presentan una alta correlación con los valores observados. 
- En el embalse seleccionado en la cuenca del Ebro, las predicciones de aportaciones son sólo capaces de discriminar el tercil superior, aunque con peores índices de verificación que en los otros embalses estudiados. Sin embargo, en el entorno de este embalse, las predicciones de precipitación demuestran pericia para discriminar tanto el carácter húmedo como el seco del próximo trimestre invernal, sobre todo el experimento que hace uso de una serie más larga del índice de nieve y que describe mejor la variabilidad de la NAO.

- La calidad de las predicciones estacionales de aportaciones y precipitación muestra ser muy sensible a la frecuencia temporal de los productos de cobertura de nieve que se usan para calcular los índices de variabilidad de la nieve en otoño usados como predictores de la NAO invernal. Los índices de variabilidad de la nieve que utilizan productos de cobertura de nieve de escala diaria producen los mejores resultados.

- Si se extiende el periodo de acumulación de las aportaciones y de precipitación para el que se hace la predicción (desde noviembre hasta marzo), la pericia de las predicciones mejora en el embalse del Ebro para las aportaciones (incluso el tercil inferior), pero disminuye en el caso de la precipitación. En el resto de embalses no se observan apenas cambios en la calidad de las predicciones ni de aportaciones ni de precipitación.

- Aunque la verificación de las predicciones probabilísticas de aportaciones y precipitación indica que existe capacidad de discriminación para las distintas categorías correspondientes a los terciles superior e inferior, también refleja la existencia de errores sistemáticos de localización y de nivel de confianza en las predicciones probabilísticas.

En general, los resultados obtenidos son muy satisfactorios teniendo en cuenta la simplicidad del método utilizado.

Entre los factores que podrían contribuir a comprender el comportamiento observado del sistema de predicción, está la distribución espacial de la correlación de la precipitación invernal con la NAO y la localización de los embalses piloto seleccionados para realizar los experimentos de validación. Los embalses piloto de las cuencas del Duero, Tajo y Guadalquivir se encuentran situados en zonas en las que la precipitación invernal está fuertemente influenciada por este patrón de variabilidad climática. Sin embargo, la cornisa norte y la franja mediterránea de España presentan valores más bajos de esta correlación. El Embalse del Ebro se encuentra en una zona de transición, lo cual explicaría al menos parcialmente la inferior pericia encontrada. Sin embargo, el hecho de que las predicciones de precipitación en el entorno de este embalse verifiquen mejor que las de aportaciones, e incluso presenten capacidad para discriminar también el tercil inferior podría indicar que existen factores relevantes que sería necesario describir en el modelo empírico para las aportaciones. Entre ellos, el manto de nieve, que no contribuye al volumen de entrada al embalse hasta su fusión, que ocurre en escalas temporales más largas que el trimestre invernal. El hecho de que la extensión del periodo de acumulación de las aportaciones desde noviembre hasta marzo produzca mejores resultados apoyaría esta hipótesis. Otra posible mejora del sistema desde el punto de vista hidrológico sería la inclusión del estado de saturación de la humedad del suelo en el otoño.

Además de los factores anteriormente mencionados, se prevén otras líneas de mejora del sistema de predicción estacional desarrollado. Entre ellas, la simulación de posibles tendencias de cambio climático y de oscilaciones a escala decadal en las aportaciones y la precipitación ligadas a la NAO en estas escalas. Otro posible perfeccionamiento del método podría proceder de una mejor representación de la incertidumbre de la predicción de la NAO, actualmente representada por una función gaussiana, o del algoritmo utilizado para generar los miembros del segundo paso de predicción.

\section{Reconocimientos}

La colaboración de los miembros del grupo de trabajo de S-ClimWaRe ha sido esencial para el desarrollo y evaluación de este modelo de predicción estacional de aportaciones a los embalses.

La Dirección General del Agua del Ministerio de Agricultura, Alimentación y Medio Ambiente ha proporcionado las series temporales de aportaciones utilizadas en este trabajo. La serie de valores mensuales del índice de la NAO empleada en este trabajo procede del Climate Prediction Center del National Weather Service de EEUU. Los productos de cobertura de nieve en rejilla usados para calcular los índices de la variabilidad de la nieve en otoño son los puestos a disposición por el National Snow and Ice Data Center de la NASA (EEUU). 


\section{Referencias}

Andreu, J., Capilla, J., Sanchis, E., 1996. AQUATOOL, a generalized decision-support system for water resources planning and operational management. J. Hydrol. 177, 269-291. doi:10.1016/00221694(95)02963-X

Brands, S., Manzanas, R., Gutiérrez, J. M., and Cohen, J., 2012. Seasonal Predictability of Wintertime Precipitation in Europe Using the Snow Advance Index. J. Climate, 25, 4023-4028. doi: 10.1175/JCLID-12-00083.1

Brown, C., Baroang, K. M., Conrad E., Lyon, B., Watkins, D., Fiondella, F., Kaheil, Y., Robertson, A., Rodriguez, J., Sheremata, M., and Ward, M. N., 2010. Managing Climate Risk in Water Supply Systems, IRI Technical Report 10-15, International Research Institute for Climate and Society, Palisades, NY, 133pp. Available online at http://iri.columbia.edu/publications/id-1048

Castro, A., Vidal, M.I., Calvo, A. I., Fernández-Raga, M., Fraile, R., 2011: May the NAO index be used to forecast rain in Spain? Atmósfera, 24 (3).

Cohen, J., and Jones, J., 2011. A new index for more accurate winter predictions. Geophys. Res. Lett., 38, L21701, doi:10.1029/2011GL049626.

Gong, G., Wang L., Condon L., Shearman, A., and Lall, U., 2010. A Simple Framework for Incorporating Seasonal Streamflow Forecasts Into Existing Water Resource Management Practices. Journal of the American Water Resources Association (JAWRA) 46(3):574-585. doi:10.1111/j.17521688.2010.00435.x

Herrera, S., Gutiérrez, J.M., Ancell, R., Pons, M.R., Frías, M.D. \& J. Fernández, 2012. Development and análisis o a 50-year high resolution daily gridded precipitation dataset over Spain (Spain0.2). Int J. Climatol. 32: 74-84. doi:10.1002/joc.2256

Hori, M. E., and Yasunari, T., 2003. NAO impact towards the springtime snow disappearance in the western Eurasian continent. Geophys. Res. Lett., 30(19), 1977, doi:10.1029/2003GL018103

Jolliffe, I. T., Stephenson, D. B., 2003. Forecast Verification. A Practitioner's Guide in Atmospheric Science. ISBN 0-471-49759-2.

Kirtman, B., Pirani, A., 2008. WCRP Position Paper on Seasonal Prediction: Report from the First WCRP Seasonal Prediction Workshop, June 4-7, 2007, Barcelona, Spain. WCRP Informal Report No. 3/2008, ICPO Publication No. 127.

Lall, U., and Sharma, A., 1996: A nearest neighbor bootstrap for resampling hydrologic time series. Water Resources Research, 32(3), 679-693.

Ogi, M., Tachibana, Y., and Yamazaki, K., 2003. Impact of the wintertime North Atlantic Oscillation (NAO) on the summertime atmospheric circulation. Geophys. Res. Lett. 30, NO 13, 1704, doi:10.1029/2003GL017280

Pouget, L., Roldán, T., Gómez, M., Cabello, A., Rodríguez-Camino, E., Navascués, B., Voces, J., Comas, P., Pastor, F., García-Gómez, M.C., Gil, J.J., Gil, D., Galván, R., 2015. Use of seasonal climate predictions in the water sector. International Conference on DROUGHT: Research and Science-Policy Interfacing (Valencia, Spain, 10-13 March 2015) https://www.crcpress.com/Drought-Research-andScience-Policy-Interfacing/Andreu-Solera-ParedesArquiola-HaroMonteagudo-van-

Lanen/9781138027794

Rodríguez-Puebla, C., Encinas, A.H., Nieto, S., Garmendia, J., 1998. Spatial and temporal patterns of annual precipitation variability over the Iberian península. International Journal of Climatology 18: 299316.

Rodríguez-Fonseca, B. and Rodríguez-Puebla, C., 2010. Teleconexiones climáticas en el entorno de la Península Ibérica. Predictabilidad y cambios esperados, In Clima en España: pasado, presente y futuro (Pérez F. Fiz and Boscolo Roberta Eds.), 53-68 pp. 
Sánchez, E., Voces, J. and Rodriguez, E., 2014. Calibration and Combination of Seasonal Forecast over Southern Europe. Catálogo de Publicaciones de la Administración General del Estado. Available online at http://goo.gl/aNScTp

Scaife, A.A., Blockley, E., Brookshaw, A., Clark, R.T., Dunstone, N., Eade, R., Fereday, D., Folland,C.K., Gordon, M., Hermanson, L., Knight, J. R., Lea, D. J., MacLachlan, C., Maidens, A., Martin, M., , Peterson, A. K., , Smith, D. , Vellinga, M., Wallace, E., Waters, J., Williams, A., 2014. Skillful long-range prediction of European and North American winters. Geophys. Res. Lett., 41, 2514-2519, doi:10.1002/2014GL059637

Troccoli, A., Harrison, M., Anderson, D.L.T., Mason, S.J., 2008. Seasonal climate: Forecasting and Managing Risk. 2008, XIV, 467 p.

Weller, R.A., Anderson, J.L., Arribas, A., Dickinson, R.E., Goddard, L., Kalnay, E., Kirtman, B., Koster, R.D., Richman, M.B., Saravanan, R., Waliser, D., Wang, B. (Committee on Assessment of Intraseasonal to Interanual Climate Prediction and Predictability), 2010. Assessment of Intraseasonal to Interannual Climate Prediction and Predictability. National Research Council. National Academies Press. ISBN: 0309-15184-8, pp 192.

Wilks, D. S., 2006. Statistical methods in the atmospheric sciences. ISBN 13:978-0-12-751966-1. 
ANEXO I

\section{Evaluación del sistema "SAl_Eurasia_weekly_ext" para dos periodos de verificación diferentes}

En este anexo se muestran los resultados obtenidos al realizar la verificación del sistema de predicción "SAl_Eurasia_weekly_ext" para el periodo DEF (Figuras 15 a 17), tanto desde un punto de vista determinista como probabilista, usando dos periodos diferentes de verificación: desde 1973 a 2013 , que corresponde a todo el periodo del que se dispone de datos de SAI semanal, y el periodo común de hindcast con los otros experimentos, desde 1999 a 2013. En estas figuras se observa que las conclusiones que se extraen del análisis del periodo común de hindcast (1999-2013) para este sistema de predicción son robustas, y se mantienen cuando se verifica un periodo más largo de 40 años. El sistema de predicción en este periodo más largo muestra incluso una mayor pericia que cuando se considera solo el más reciente.

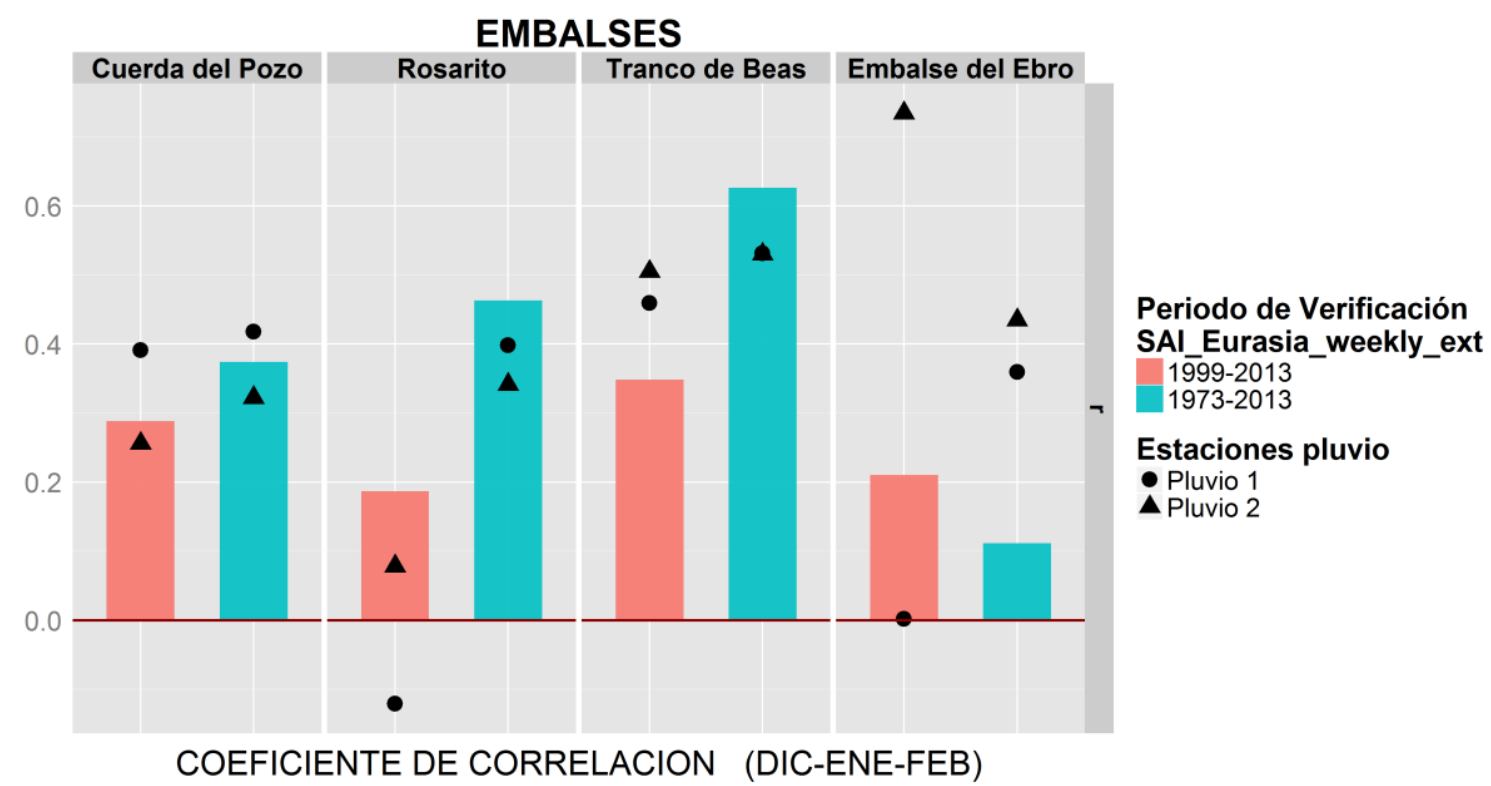

Figura 15. Coeficiente de correlación para el periodo DEF por embalses, entre observaciones y la media del ensemble del sistema de predicción "SAI_Eurasia_weekly_ext" para dos periodos de verificación (1999-2013 y 1973-2013). Los valores mostrados en forma de barras de colores en función del sistema de predicción, corresponden a los valores obtenidos para las aportaciones a cada embalse y los valores obtenidos para la precipitación medida en las diferentes estaciones pluviométricas se muestran con puntos en forma de círculos y triángulos en la vertical del de la aportación correspondiente. 


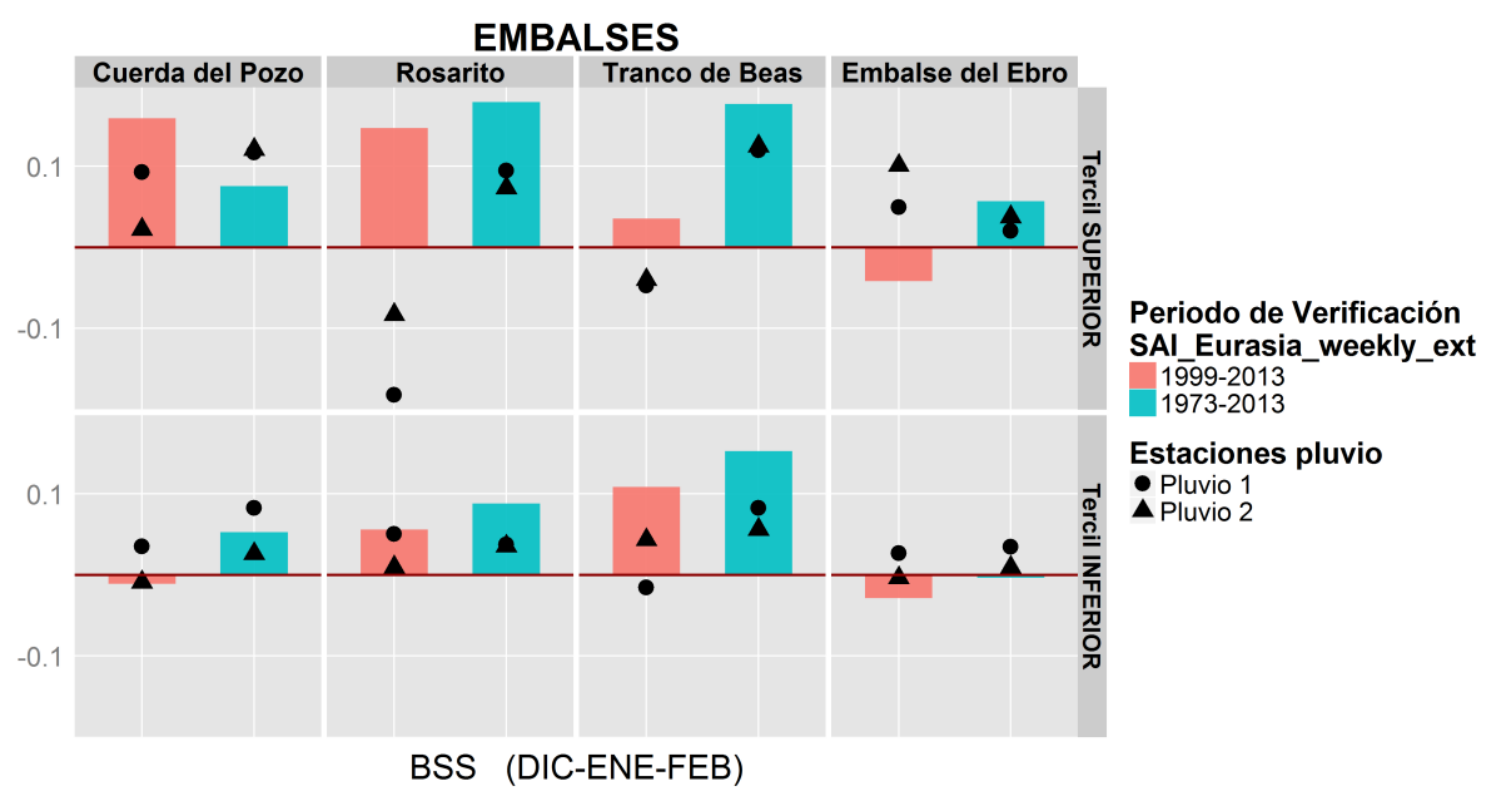

Figura 16. BSS del sistema de predicción SAI Eurasia weekly ext para valores de aportaciones/precipitación en DEF por encima y por debajo de los valores normales en dos periodos de verificación diferentes (1999-2013 y 1973-2013). Los valores mostrados en forma de barras de colores corresponden a las aportaciones a cada embalse. Los valores de este índice de verificación obtenidos para la precipitación en las diferentes estaciones pluviométricas se muestran con puntos en forma de círculos y triángulos en la vertical del de la aportación correspondiente.

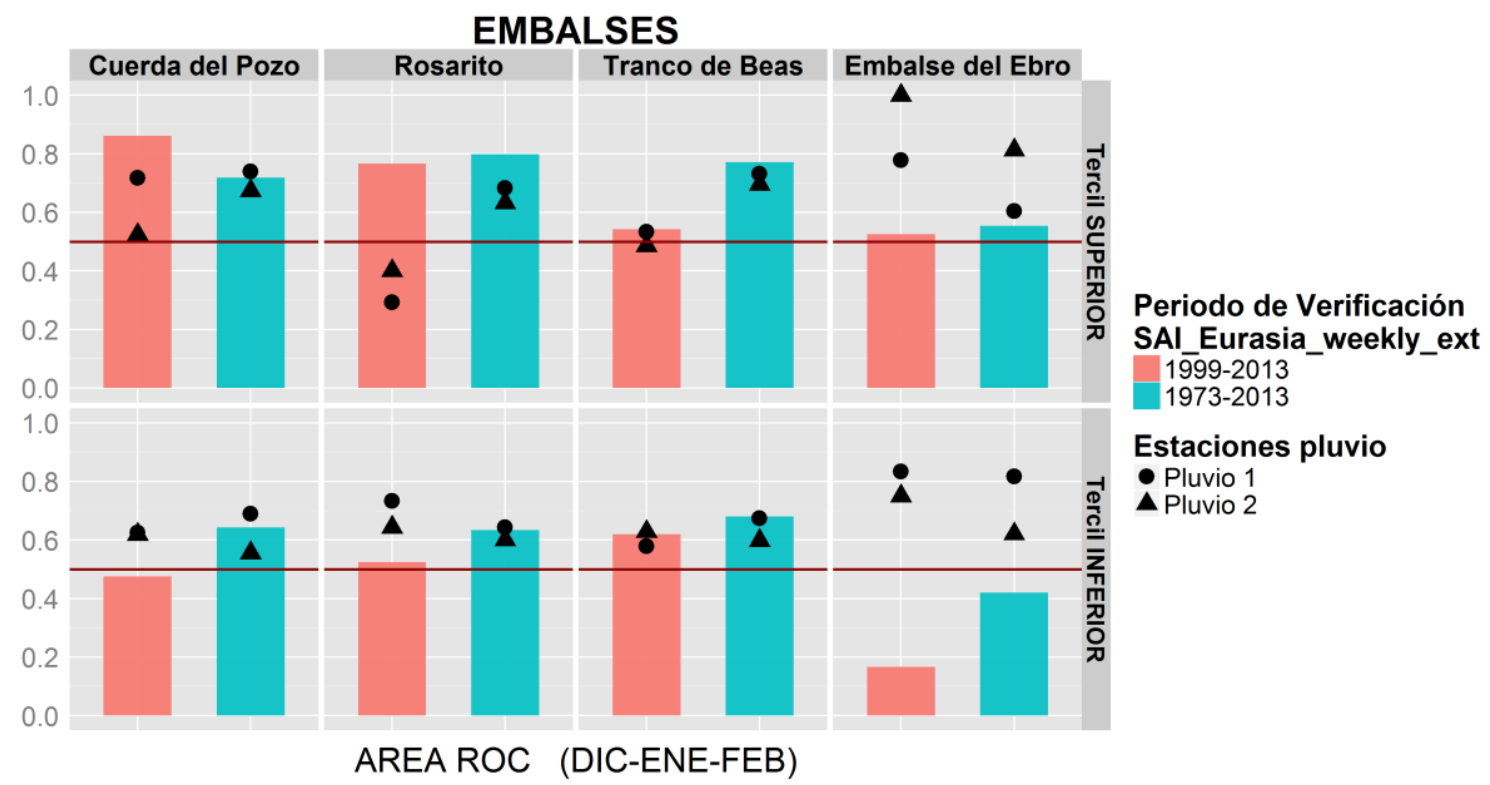

Figura 17. Área ROC del sistema de predicción SAI_Eurasia_weekly_ext para valores de aportaciones/precipitación en DEF por encima y por debajo de los valores normales en dos periodos de verificación diferentes (1999-2013 y 1973-2013). Los valores mostrados en forma de barras de colores corresponden a las aportaciones a cada embalse. Los valores de este índice de verificación obtenidos para la precipitación en las diferentes estaciones pluviométricas se muestran con puntos en forma de círculos y triángulos en la vertical del de la aportación correspondiente. 